\title{
ON HOPF'S LEMMA AND THE STRONG MAXIMUM PRINCIPLE
}

\author{
S. BERTONE, A. CELLINA, AND E. M. MARCHINI \\ AbStract. In this paper we consider Hopf's Lemma and the Strong Maximum \\ Principle for supersolutions to

$$
\sum_{i=1}^{N} g_{i}\left(u_{x_{i}}^{2}\right) u_{x_{i} x_{i}}=0
$$ \\ under suitable hypotheses that allow $g_{i}$ to assume value zero at zero.
}

\section{INTRODUCTION}

Let $\Omega \subset \mathbb{R}^{N}$ be a connected, open and bounded set; we call $\Omega$ regular if for every $z \in \partial \Omega$, there exists a tangent plane, continously depending on $z$. We say that $\Omega$ satisfies the interior ball condition at $z$ if there exists an open ball $B \subset \Omega$ with $z \in \partial B$. On $\Omega$, consider the operator

$$
F(u)=\sum_{i=1}^{N} g_{i}\left(u_{x_{i}}^{2}\right) u_{x_{i} x_{i}},
$$

where $g_{i}:[0,+\infty) \rightarrow[0,+\infty)$ are continuous functions.

When $F$ is elliptic, two classical results hold.

Hopf's Lemma:

Let $\Omega$ be regular, let $u$ be such that $F(u) \leq 0$ on $\Omega$. Suppose that there exists $z \in \partial \Omega$ such that

$$
u(z)<u(x), \quad \text { for all } x \text { in } \Omega .
$$

If, in addition, $\Omega$ satisfies the interior ball condition at $z$, we have

$$
\frac{\partial u}{\partial \nu}(z)<0
$$

where $\nu$ is the outer unit normal to $B$ at $z$.

The Strong Maximum Principle:

Let $u$ be such that $F(u) \leq 0$ on $\Omega$, then if $u$ it attains minimum in $\Omega$, it is a constant.

In 1927 Hopf proved the Strong Maximum Principle in the case of second order elliptic partial differential equations, by applying a comparison technique, see [1]. For the class of quasilinear elliptic problems, many contributions have been given, to extend the validity of the previous results, as in [1, 5, 6, 8, 9, 10, 12, 13, 14, 15, [16. 18.

Key words and phrases. Strong maximum principle. 
In the case in equation (11) we have $g_{i} \equiv 1$, for every $i$, then $F(u)=\Delta u$, and we find the classical problem of the Laplacian, see [7, 9].

On the other hand, when there exists $i \in\{1, \ldots, N\}$ such that $g_{i} \equiv 0$ on an interval $I=[0, T] \subset \mathbb{R}$, the Strong Maximum Principle does not hold. Indeed, in this case, it is always possible to define a function $u$ assuming minimum in $\Omega$ and such that $\sum_{i=1}^{N} g_{i}\left(u_{x_{i}}^{2}\right) u_{x_{i} x_{i}}=0$. For instance, let $g_{N}(t)=0$, for every $t \in[0,2]$. The function

$$
u\left(x_{1}, \ldots, x_{N}\right)=\left\{\begin{array}{ll}
-\left(x_{N}^{2}-1\right)^{4} & \text { if }-1 \leq x_{N} \leq 1 \\
0 & \text { otherwise }
\end{array},\right.
$$

satisfies (1) in $\mathbb{R}^{N}$.

We are interested in the case when $0 \leq g_{i}(t) \leq 1$ and it does not exist $i$ such that $g_{i} \equiv 0$ on an interval. Since $g_{i}$ could assume value zero, the equation (11) is non elliptic.

The results known so far, for the validity of Hopf's Lemma and of the Strong Maximum Principle, suggest that, for possibly non elliptic equations, but arising from a functional having rotational symmetry, this validity shall depend only on the behaviour of the functions $g_{i}$ near zero, see 4 .

In this paper, we prove, in section 3 , a sufficient condition for the validity of the Hopf's Lemma and of the Strong Maximum Principle; a remarkable feature of this condition is that it concerns only the behaviour of the function $g_{i}(t)$ that goes fastest to zero, as $t$ goes to zero. Hopf's lemma and the Strong Maximum Principle are essentially the same result as long as we can build subsolutions whose level lines can have arbitrarily large curvature. This need not be always possible for problems not possessing rotational symmetry. This difficulty will be evident in sections 4 and 5. In these sections, a more restricted class of equations is considered, namely when all the functions $g_{i}$, for $i=1, \ldots, N-1$, are 1 and only $g_{N}$ is allowed to go to zero. In this simpler class of equations we are able to show that the condition

$$
\lim _{t \rightarrow 0^{+}} \frac{\left(g_{N}(t)\right)^{3 / 2}}{t g_{N}^{\prime}(t)}>0
$$

is at once necessary for the validity of Hopf's Lemma and sufficient for the validity of the Strong Maximum Principle.

\section{Preliminary Results}

We impose the following local assumptions.

Assumptions (L):

There exists $\bar{t}>0$ such that:

i) on $[0, \bar{t}]$, for every $i=1, \ldots, N-1$,

$$
0 \leq g_{N}(t) \leq g_{i}(t) \leq 1
$$

ii) $g_{N}$ is continuous on $[0, \bar{t}]$; positive and differentiable on $(0, \bar{t}]$;

iii) on $(0, \bar{t}]$, the function $t \rightarrow g_{N}(t)+g_{N}^{\prime}(t) t$ is non decreasing. 
Notice that, in case ii) above is violated, the Strong Maximum Principle does not hold; and that condition iii) above includes the case of the Laplacian, $g_{i}(t) \equiv 1$; and, finally, that under these assumptions, $g_{i}$ could assume value zero at most for $t=0$.

Moreover, we can consider the equation

$$
\sum_{i=1}^{N} g_{i}\left(u_{x_{i}}^{2}\right) u_{x_{i} x_{i}}=0
$$

as the Euler-Lagrange equation associated to the functional

$$
J(u)=\int_{\Omega} L(\nabla u) d \Omega=\int_{\Omega} \frac{1}{2}\left(\sum_{i=1}^{N} f_{i}\left(u_{x_{i}}^{2}\right) u_{x_{i}}^{2}\right) d \Omega,
$$

where $L(\nabla u)$ is strictly convex in $\left\{\left(u_{x_{1}}, \ldots, u_{x_{N}}\right): u_{x_{i}}^{2} \leq \bar{t}\right.$, for every $\left.i=1, \ldots, N\right\}$. Indeed, fix $i$. Let $f_{i}$ be a solution to the differential equation

$$
g_{i}(t)=f_{i}(t)+5 t f_{i}^{\prime}(t)+2 t^{2} f_{i}^{\prime \prime}(t)
$$

for $t \in[0, \bar{t}]$. Since

$$
\frac{\partial^{2} L}{\partial u_{x_{i}}^{2}}\left(u_{x_{i}}^{2}\right)=f_{i}\left(u_{x_{i}}^{2}\right)+5 u_{x_{i}}^{2} f_{i}^{\prime}\left(u_{x_{i}}^{2}\right)+2 u_{x_{i}}^{4} f_{i}^{\prime \prime}\left(u_{x_{i}}^{2}\right)=g_{i}\left(u_{x_{i}}^{2}\right),
$$

we have that

$$
\operatorname{div} \nabla_{\nabla u} L(\nabla u)=\sum_{i=1}^{N} \frac{\partial^{2} L}{\partial u_{x_{i}}^{2}} u_{x_{i} x_{i}}=\sum_{i=1}^{N} g_{i}\left(u_{x_{i}}^{2}\right) u_{x_{i} x_{i}}
$$

Moreover, the strict convexity of $L(\nabla u)$ in $\left\{\left(u_{x_{1}}, \ldots, u_{x_{N}}\right): u_{x_{i}}^{2} \leq \bar{t}\right.$, for every $i=$ $1, \ldots, N\}$ follows by the fact that $g_{i}$ is positive in $(0, \bar{t}]$.

Since we will need general comparison theorems that depend on the global properties of the solutions, i.e. on their belonging to a Sobolev space, we will need also a growth assumption on $g_{i}$ (assumption $(G)$ ) to insure these properties of the solutions.

Assumption ( $G)$ :

Each function $f_{i}$ as defined in (2), is bounded and $f_{i}\left(u_{x_{i}}^{2}\right) u_{x_{i}}^{2}$ is strictly convex.

Any function $g_{i}$ satisfying assumptions $(L)$ on $[0, \bar{t}]$ can be extended so as to satisfy assumption $(G)$ on $[0,+\infty)$. In fact, it is enough to extend $g_{i}$ to $(\bar{t},+\infty)$ by setting $g_{i}(t)=f_{i}(\bar{t})$, for $t>\bar{t}$.

Definition 1. Let $\Omega$ be open, and let $u \in W^{1,2}(\Omega)$. The map $u$ is a weak solution to the equation $F(u)=0$ if, for every $\eta \in C_{0}^{\infty}(\Omega)$,

$$
\int_{\Omega}\langle\nabla L(\nabla u(x)), \nabla \eta(x)\rangle d x=0 .
$$

$u$ is a weak subsolution $(F(u) \geq 0)$ if, for every $\eta \in C_{0}^{\infty}(\Omega), \eta \geq 0$,

$$
\int_{\Omega}\langle\nabla L(\nabla u(x)), \nabla \eta(x)\rangle d x \leq 0 .
$$


$u$ is a weak supersolution $(F(u) \leq 0)$ if, for every $\eta \in C_{0}^{\infty}(\Omega), \eta \geq 0$,

$$
\int_{\Omega}\langle\nabla L(\nabla u(x)), \nabla \eta(x)\rangle d x \geq 0 .
$$

We say that a function $w \in W^{1,2}(\Omega)$ is such that $w_{\mid \partial \Omega} \leq 0$ if $w^{+} \in W_{0}^{1,2}(\Omega)$.

The growth assumption $(G)$ assures that, if $u \in W^{1,2}(\Omega)$, then $\nabla L(\nabla u(x)) \in$ $L^{2}(\Omega)$. The strict convexity of $L$ implies the following comparison lemma.

Lemma 1. Let $\Omega$ be a open and bounded set, let $v \in W^{1,2}(\Omega)$ be a subsolution and let $u \in W^{1,2}(\Omega)$ be a supersolution to the equation $F(u)=0$. If $v_{\mid \partial \Omega} \leq u_{\mid \partial \Omega}$, then $v \leq u$ a.e. in $\Omega$.

We wish to express the operator

$$
F(v)=\sum_{i=1}^{N} g_{i}\left(v_{x_{i}}^{2}\right) v_{x_{i} x_{i}}
$$

in polar coordinates. Set

$$
\left\{\begin{array}{l}
x_{1}=\rho \cos \theta_{N-1} \ldots \cos \theta_{2} \cos \theta_{1} \\
x_{2}=\rho \cos \theta_{N-1} \ldots \cos \theta_{2} \sin \theta_{1} \\
\ldots \\
x_{N}=\rho \sin \theta_{N-1}
\end{array}\right.
$$

so that

$$
v_{x_{i}}=v_{\rho} \frac{x_{i}}{\rho} \quad \text { and } \quad v_{x_{i} x_{i}}=v_{\rho \rho}\left(\frac{x_{i}}{\rho}\right)^{2}+\frac{v_{\rho}}{\rho}\left[1-\left(\frac{x_{i}}{\rho}\right)^{2}\right] .
$$

When $v$ is a radial function, $F$ reduces to

$$
\begin{gathered}
F(v)=\sum_{i=1}^{N} g_{i}\left(v_{\rho}^{2}\left(\frac{x_{i}}{\rho}\right)^{2}\right)\left[v_{\rho \rho}\left(\frac{x_{i}}{\rho}\right)^{2}+\frac{v_{\rho}}{\rho}\left(1-\left(\frac{x_{i}}{\rho}\right)^{2}\right)\right]= \\
v_{\rho \rho} \sum_{i=1}^{N} g_{i}\left(v_{\rho}^{2}\left(\frac{x_{i}}{\rho}\right)^{2}\right)\left(\frac{x_{i}}{\rho}\right)^{2}+\frac{v_{\rho}}{\rho} \sum_{i=1}^{N} g_{i}\left(v_{\rho}^{2}\left(\frac{x_{i}}{\rho}\right)^{2}\right)\left(1-\left(\frac{x_{i}}{\rho}\right)^{2}\right) .
\end{gathered}
$$

In general, we do not expect that the equation $F(v)=0$ admits radial solutions. However we will use the expression of $F$ valid for radial functions in order to reach our results.

The following technical lemmas will be used later.

Lemma 2. Let $n=2, \ldots, N$ and set

$$
h_{n}(a)=g_{N}\left(\frac{t(1-a)}{n-1}\right)(1-a)+g_{N}(t a) a .
$$

For every $0<t \leq \bar{t}$ ( $\bar{t}$ defined in assumptions $(L)), h_{n}(a) \geq h_{n}(1 / n)$, for every a in $[0,1]$.

Proof. Since, on $(0, \bar{t}]$, the function $t \rightarrow g_{N}(t)+g_{N}^{\prime}(t) t$ is non decreasing, we have that

$$
h_{n}^{\prime}(a)=-g_{N}\left(\frac{t(1-a)}{n-1}\right)-g_{N}^{\prime}\left(\frac{t(1-a)}{n-1}\right) \frac{t(1-a)}{n-1}+g_{N}(t a)+g_{N}^{\prime}(t a) t a \geq 0
$$


if and only if $a \geq 1 / n$, so that $h_{n}(a) \geq h_{n}(1 / n)$, for every $a \in[0,1]$.

Lemma 3. For every $0<t \leq \bar{t}$ ( $\bar{t}$ defined in assumptions $(L)$ ), we have that

$$
\sum_{i=1}^{N} g_{N}\left(t\left(\frac{x_{i}}{\rho}\right)^{2}\right)\left(\frac{x_{i}}{\rho}\right)^{2} \geq g_{N}\left(\frac{t}{N}\right) .
$$

Proof. We prove the claim by induction on $N$.

Let $N=2$. Set $a=\sin ^{2} \theta_{1}$. Applying Lemma 2 we obtain that

$$
\begin{gathered}
g_{N}\left(t\left(\frac{x_{1}}{\rho}\right)^{2}\right)\left(\frac{x_{1}}{\rho}\right)^{2}+g_{N}\left(t\left(\frac{x_{2}}{\rho}\right)^{2}\right)\left(\frac{x_{2}}{\rho}\right)^{2}= \\
g_{N}(t(1-a))(1-a)+g_{N}(t a) a \geq g_{N}\left(\frac{t}{2}\right) .
\end{gathered}
$$

Suppose that the claim is true for $N$ - 1, i.e.

$$
\sum_{i=1}^{N-1} g_{N}\left(t\left(\frac{x_{i}}{\rho}\right)^{2}\right)\left(\frac{x_{i}}{\rho}\right)^{2} \geq g_{N}\left(\frac{t}{N-1}\right) \text {. }
$$

Let us prove it for $N$. Set

$$
\left\{\begin{array}{l}
y_{1}=\rho \cos \theta_{N-2} \ldots \cos \theta_{2} \cos \theta_{1} \\
y_{2}=\rho \cos \theta_{N-2} \ldots \cos \theta_{2} \sin \theta_{1} \\
\cdots \\
y_{N-1}=\rho \sin \theta_{N-2}
\end{array}\right.
$$

and set $a=\sin ^{2} \theta_{N-1}$. Applying Lemma 2 we obtain that

$$
\begin{gathered}
\sum_{i=1}^{N} g_{N}\left(t\left(\frac{x_{i}}{\rho}\right)^{2}\right)\left(\frac{x_{i}}{\rho}\right)^{2}= \\
\sum_{i=1}^{N-1} g_{N}\left(t\left(\frac{y_{i}}{\rho}\right)^{2}(1-a)\right)\left(\frac{y_{i}}{\rho}\right)^{2}(1-a)+g_{N}(t a) a \geq \\
g_{N}\left(\frac{t(1-a)}{N-1}\right)(1-a)+g_{N}(t a) a \geq g_{N}\left(\frac{t}{N}\right),
\end{gathered}
$$

and the claim is proved.

\section{A sufficient CONDition for the VALidity of Hopf's Lemma AND OF} the Strong Maximum Principle

Consider the improper Riemann integral

$$
\int_{0}^{\xi} \frac{g_{N}\left(\zeta^{2} / N\right)}{\zeta} d \zeta=\lim _{\widehat{\xi} \rightarrow 0} \int_{\widehat{\xi}}^{\xi} \frac{g_{N}\left(\zeta^{2} / N\right)}{\zeta} d \zeta
$$

as an extended valued function $G$,

$$
G(\xi)=\int_{0}^{\xi} \frac{g_{N}\left(\zeta^{2} / N\right)}{\zeta} d \zeta
$$

where we mean that $G(\xi) \equiv+\infty$ whenever the integral diverges. 
We wish to prove the following lemma.

Lemma 4 (Hopf's Lemma). Let $\Omega \subset \mathbb{R}^{N}$ be a connected, open and bounded set. Let $u \in W^{1,2}(\Omega) \cap C(\bar{\Omega})$ be a weak solution to

$$
\sum_{i=1}^{N} g_{i}\left(u_{x_{i}}^{2}\right) u_{x_{i} x_{i}} \leq 0 .
$$

In addition to the assumptions $(L)$ and $(G)$ on $g_{i}$, assume that $G(\xi) \equiv+\infty$. Suppose that there exists $z \in \partial \Omega$ such that

$$
u(z)<u(x), \quad \text { for all } x \text { in } \Omega
$$

and that $\Omega$ satisfies the interior ball condition at $z$. Then

$$
\frac{\partial u}{\partial \nu}(z)<0
$$

where $\nu$ is the outer unit normal to $B$ at $z$.

As an example of an equation satisfying the assumptions of the theorem above, consider the Laplace equation $\Delta u=0$. The functions $g_{i} \equiv 1$ satisfy the assumptions $(L)$ and $(G)$, and

$$
G(\xi)=\int_{0}^{\xi} \frac{1}{\zeta} d \zeta=+\infty .
$$

Another example is obtained setting

$$
g_{N}(t)=\frac{1}{|\ln (t)|}
$$

for $0 \leq t \leq 1 / e$. The assumptions $(L)$ and $(G)$ are satisfied; moreover, for $0 \leq$ $\xi^{2} / N \leq 1 / e$,

$$
G(\xi)=\int_{0}^{\xi} \frac{d \zeta}{\zeta\left|\ln \left(\zeta^{2} / N\right)\right|}=+\infty
$$

Proof of Lemma 4. a) Assume that $u(z)=0$ and that $B=B(O, r)$. We prove the claim by contradiction. Suppose that

$$
\frac{\partial u}{\partial \nu}(z) \geq 0
$$

where $\nu$ is the outer unit normal to $B$ at $z$. Let $\epsilon=\min \{u(x): x \in \overline{B(O, r / 2)}\}$; we have that $\epsilon>0$. Set

$$
\omega=B(O, r) \backslash \overline{B(O, r / 2)} \text {. }
$$

b) We seek a radial function $v \in W^{1,2}(\omega) \cap C(\bar{\omega})$ satisfying

$$
\begin{cases}v \text { is a weak solution to } F(v) \geq 0 & \text { in } \omega \\ v>0 & \text { in } \omega \\ v=0 & \text { in } \partial B(O, r) \\ v \leq \epsilon & \text { in } \partial B(O, r / 2) \\ v_{\rho}(z)<0 . & \end{cases}
$$


Consider the Cauchy problem

$$
\left\{\begin{array}{l}
\zeta^{\prime}=-\frac{N-1}{\rho} \frac{\zeta}{g_{N}\left(\zeta^{2} / N\right)} \\
\zeta(r / 2)=-\frac{\epsilon}{r} .
\end{array}\right.
$$

There exists a unique local solution $\zeta$ of (4), such that

$$
\int_{-\frac{\epsilon}{r}}^{\zeta(\rho)} \frac{g_{N}\left(\zeta^{2} / N\right)}{\zeta} d \zeta=\int_{r / 2}^{\rho}-\frac{N-1}{s} d s=-(N-1) \ln \left(\frac{2 \rho}{r}\right) .
$$

We claim that $\zeta$ is defined in $[r / 2,+\infty)$. Indeed, suppose that $\zeta$ is defined in $[r / 2, \tau)$, with $\tau<+\infty$. Since $\zeta^{\prime}>0, \zeta$ is an increasing function, so that $\tau<+\infty$ if and only if $\lim _{\rho \rightarrow \tau} \zeta(\rho)=0$. But

$$
-\infty=\lim _{\rho \rightarrow \tau} \int_{-\frac{\epsilon}{r}}^{\zeta(\rho)} \frac{g_{N}\left(\zeta^{2} / N\right)}{\zeta} d \zeta=\lim _{\rho \rightarrow \tau}-(N-1) \ln \left(\frac{2 \rho}{r}\right),
$$

a contradiction. Hence, the solution $\zeta$ of (4) is defined in $[r / 2,+\infty)$.

Setting $v_{\rho}=\zeta$, since, for every $\rho \in(r / 2, r)$,

$$
-\frac{\epsilon}{r}<v_{\rho}(\rho)<0
$$

we have that the function

$$
v(\rho)=\int_{r}^{\rho} v_{\rho}(s) d s
$$

solves the problem

$$
v_{\rho \rho} g_{N}\left(\frac{v_{\rho}^{2}}{N}\right)+\frac{v_{\rho}}{\rho}(N-1)=0,
$$

in particular, $v(\rho)>0$ and $v_{\rho}(\rho)<0$, for every $\rho \in(r / 2, r), v(r)=0$ and $v(r / 2) \leq$ $\epsilon$.

Since $v_{\rho \rho} \geq 0$ and $-\sqrt{\bar{t}} \leq v_{\rho} \leq 0$, for every $\rho \in(r / 2, r)$, by the hypotheses on $g_{i}$ and by Lemma 3 we have that

$$
\begin{gathered}
F(v)=v_{\rho \rho} \sum_{i=1}^{N} g_{i}\left(v_{\rho}^{2}\left(\frac{x_{i}}{\rho}\right)^{2}\right)\left(\frac{x_{i}}{\rho}\right)^{2}+\frac{v_{\rho}}{\rho} \sum_{i=1}^{N} g_{i}\left(v_{\rho}^{2}\left(\frac{x_{i}}{\rho}\right)^{2}\right)\left(1-\left(\frac{x_{i}}{\rho}\right)^{2}\right) \geq \\
v_{\rho \rho} \sum_{i=1}^{N} g_{N}\left(v_{\rho}^{2}\left(\frac{x_{i}}{\rho}\right)^{2}\right)\left(\frac{x_{i}}{\rho}\right)^{2}+\frac{v_{\rho}}{\rho} \sum_{i=1}^{N}\left(1-\left(\frac{x_{i}}{\rho}\right)^{2}\right)= \\
v_{\rho \rho} \sum_{i=1}^{N} g_{N}\left(v_{\rho}^{2}\left(\frac{x_{i}}{\rho}\right)^{2}\right)\left(\frac{x_{i}}{\rho}\right)^{2}+\frac{v_{\rho}}{\rho}(N-1) \geq v_{\rho \rho} g_{N}\left(\frac{v_{\rho}^{2}}{N}\right)+\frac{v_{\rho}}{\rho}(N-1) .
\end{gathered}
$$

The function $v$ solves (3), indeed, $v$ is in $C^{2}(\bar{\omega})$ and it is such that $F(v) \geq 0$ and $v>0$ in $\omega, v(r)=0, v(r / 2) \leq \epsilon$ and $v_{\rho}(z)<0$.

c) Since $u, v \in W^{1,2}(\omega) \cap C(\bar{\omega}), v$ is a weak subsolution and $u$ is a weak solution to $F(u)=0$, and $v_{\mid \partial \omega} \leq u_{\mid \partial \omega}$, applying Lemma 1 we obtain that $v \leq u$ in $\omega$. From

$$
v_{\rho}(z)=\frac{\partial v}{\partial \nu}(z)<\frac{\partial u}{\partial \nu}(z)
$$

it follows that there exists $x^{0} \in \omega$ such that $v\left(x^{0}\right)>u\left(x^{0}\right)$, a contradiction. 
From Hopf's Lemma we derive:

Theorem 1 (Strong Maximum Principle). Let $\Omega \subset \mathbb{R}^{N}$ be a connected, open and bounded set. Let $u \in W^{1,2}(\Omega) \cap C(\bar{\Omega})$ be a weak supersolution to

$$
\sum_{i=1}^{N} g_{i}\left(u_{x_{i}}^{2}\right) u_{x_{i} x_{i}}=0 .
$$

In addition to the assumptions $(L)$ and $(G)$ on $g_{i}$, assume that $G(\xi) \equiv+\infty$. Then, if $u$ attains its minimum in $\Omega$, it is a constant.

Proof. a) Assume $\min _{\Omega} u=0$ and set $\mathcal{C}=\{x \in \Omega: u(x)=0\}$. By contradiction, suppose that the open set $\Omega \backslash \mathcal{C} \neq \emptyset$.

b) Since $\Omega$ is a connected set, there exist $s \in \mathcal{C}$ and $R>0$ such that $B(s, R) \subset \Omega$ and $B(s, R) \cap(\Omega \backslash \mathcal{C}) \neq \emptyset$. Let $p \in B(s, R) \cap(\Omega \backslash \mathcal{C})$. Consider the line $\overline{p s}$. Moving $p$ along this line, we can assume that $B(p, d(p, \mathcal{C})) \subset(\Omega \backslash \mathcal{C})$ and that there exists one point $z \in \mathcal{C}$ such that $d(p, \mathcal{C})=d(p, z)$. Set $r=d(p, \mathcal{C})$. W.l.o.g. suppose that $p=O$.

c) The set $\Omega \backslash \mathcal{C}$ satisfies the interior ball condition at $z$, hence Hopf's Lemma implies

$$
\frac{\partial u}{\partial \nu}(z)<0
$$

But this is a contradiction: since $u$ attains minimum at $z \in \Omega$, we have that $D u(z)=0$.

\section{A necessary Condition for the Validity of Hopf's Lemma}

In this and the following section we consider the operator

$$
F(u)=\sum_{i=1}^{N-1} u_{x_{i} x_{i}}+g\left(u_{x_{N}}^{2}\right) u_{x_{N} x_{N}},
$$

We wish to provide a necessary condition for the validity of Hopf's Lemma in a class of non elliptic equations.

Consider the case

$$
G(\xi)=\int_{0}^{\xi} \frac{g\left(\zeta^{2} / N\right)}{\zeta} d \zeta<+\infty .
$$

Theorem 2. Consider the operator (5), where g satisfies assumptions $(L)$ and $(G)$, and on $(0, \bar{t}]$ ( $\bar{t}$ defined in assumptions $(L))$,

$$
g^{\prime}(t)>0 \quad \text { and } \quad g(t)+g^{\prime}(t) t \leq 1 .
$$

If

$$
\lim _{t \rightarrow 0^{+}} \frac{(g(t))^{3 / 2}}{t g^{\prime}(t)}=0
$$


then there exist: an open regular region $\Omega \subset \mathbb{R}^{N} ;$ a radial function $u \in C^{2}(\Omega)$ such that $F(u) \leq 0$ in $\Omega$ and a point $z \in \partial \Omega$ such that $u(z)=0, u(z) \leq u(x)$ for every $x \in \Omega$ and

$$
\frac{\partial u}{\partial \nu}(z)=0
$$

where $\nu$ is the outer unit normal to $\Omega$ at $z$.

If, in addition, we assume that

$$
\frac{g(t)}{t g^{\prime}(t)} \quad \text { is bounded in } \quad(0, \bar{t}]
$$

then $\Omega$ satisfies the interior ball condition at $z$.

Remark 1. When $\lim _{t \rightarrow 0^{+}} g^{\prime}(t) t$ exists, it follows that

$$
\lim _{t \rightarrow 0^{+}} \frac{(g(t))^{3 / 2}}{t g^{\prime}(t)}
$$

exists, and that

$$
g(t)+g^{\prime}(t) t \leq 1, \text { on }(0, \bar{t}]
$$

Indeed, we have that

$$
\lim _{t \rightarrow 0^{+}}\left(g(t)+g^{\prime}(t) t\right)=0 .
$$

Otherwise, there exists $K>0$ such that, when $0<t \leq \bar{t}, g^{\prime}(t) t \geq K$, so that

$$
g(t) t=\int_{0}^{t}\left(g(s)+g^{\prime}(s) s\right) d s \geq K t,
$$

and $g(t) \geq K$. From

$$
\int_{0}^{\xi^{2}} \frac{g(t)}{t} d t<+\infty
$$

it follows that $\lim _{t \rightarrow 0^{+}} g(t)=0$, a contradiction.

The map

$$
g(t)=\frac{1}{|\ln (t)|^{k}}
$$

with $k>2$, for $0 \leq t \leq 1 / e$, satisfies the assumption

$$
\lim _{t \rightarrow 0^{+}} \frac{(g(t))^{3 / 2}}{t g^{\prime}(t)}=0
$$

The following lemma is instrumental to the proofs of the main results.

Lemma 5. Let $g$ satisfies assumptions $(L)$ and $(G)$. Suppose that for every $0<$ $t \leq \bar{t}$

$$
g^{\prime}(t) \geq 0 \quad \text { and } \quad g(t)+g^{\prime}(t) t \leq 1
$$

Set

$$
k_{1}(a)=(1-a)+a g(t a) \quad \text { and } \quad k_{2}(a)=-a-(1-a) g(t a)
$$

For every $0<t \leq \bar{t}(\bar{t}$ defined in assumptions $(L)), k_{1}$ and $k_{2}$ are non increasing in $[0,1]$. 
Proof. Since, for every $0<t \leq \bar{t}$,

$$
g^{\prime}(t) \geq 0 \quad \text { and } \quad g(t)+g^{\prime}(t) t \leq 1,
$$

we have that, for every $0 \leq a \leq 1$,

$$
k_{1}^{\prime}(a)=-1+g(t a)+g^{\prime}(t a) t a \leq 0
$$

and

$$
k_{2}^{\prime}(a)=-1+g(t a)-(1-a) g^{\prime}(t a) t=-1+g(t a)+g^{\prime}(t a) t a-g^{\prime}(t a) t \leq 0 .
$$

Proof of Theorem 2 a) Let $v$ be a radial function. Setting $a=\sin ^{2} \theta_{N-1}$, (5) reduces to

$$
F(v)=v_{\rho \rho}\left(1-a+a g\left(v_{\rho}^{2} a\right)\right)+\frac{v_{\rho}}{\rho}\left(N-2+a+(1-a) g\left(v_{\rho}^{2} a\right)\right) .
$$

Let $a=1$, we seek a solution to

$$
v_{\rho \rho} g\left(v_{\rho}^{2}\right)+(N-1) \frac{v_{\rho}}{\rho}=0
$$

such that $v_{\rho}(R(1)+1)=0$ and $v_{\rho}(\rho)<0$, for every $\rho \in[2, R(1)+1)$. Consider the Cauchy problem

$$
\left\{\begin{array}{l}
\zeta^{\prime}=-\frac{N-1}{\rho} \frac{\zeta}{g\left(\zeta^{2}\right)} \\
\zeta(2)=-1 .
\end{array}\right.
$$

We are interested in a negative solution $\zeta$. Define $R(1)$ to be the unique positive real solution to

$$
G(-1)-(N-1) \ln \left(\frac{R(1)+1}{2}\right)=0,
$$

i.e.

$$
R(1)=2 e^{\frac{G(-1)}{N-1}}-1 .
$$

The solution $\zeta$ of (8), satisfies

$$
G(\zeta(\rho))-G(-1)=\int_{\zeta(2)}^{\zeta(\rho)} \frac{g\left(t^{2}\right)}{t} d t=\int_{2}^{\rho}-\frac{N-1}{s} d s=-(N-1) \ln \left(\frac{\rho}{2}\right) .
$$

Then, for every $\rho \in(2, R(1)+1), G(\zeta(\rho))>0$ and $\zeta(\rho)<0$, while $\zeta(R(1)+1)=0$. Setting $v_{\rho}(\rho)=\zeta(\rho)$ and

$$
v(\rho)=\int_{R(1)+1}^{\rho} v_{\rho}(s) d s,
$$

we obtain that $v$ solves (77) and, for every $\rho \in(2, R(1)+1)$,

$$
v_{\rho}(\rho)<v_{\rho}(R(1)+1)=0 \quad \text { and } \quad v(\rho)>v(R(1)+1)=0 .
$$

b) Set, for $\rho \in(1, R(1)], u(\rho)=v(\rho+1)$. Since, for the function $v$, we have

$$
v_{\rho \rho}(\rho) g\left(v_{\rho}^{2}(\rho)\right)+(N-1) \frac{v_{\rho}(\rho)}{\rho}=0,
$$

at $\rho+1$ we obtain

$$
u_{\rho \rho}(\rho) g\left(u_{\rho}^{2}(\rho)\right)+(N-1) \frac{u_{\rho}(\rho)}{\rho+1}=0 .
$$


This equality yields, for $\rho \in(1, R(1))$,

$$
u_{\rho \rho} g\left(u_{\rho}^{2}\right)+(N-1) \frac{u_{\rho}}{\rho}=-(N-1) u_{\rho}\left(\frac{1}{\rho+1}-\frac{1}{\rho}\right)<0 .
$$

c) Let $1 / 2<a<1$. We wish to find $R(a) \leq R(1)$ such that $u$ is a solution to

$$
F(u)=u_{\rho \rho}\left(1-a+a g\left(u_{\rho}^{2} a\right)\right)+\frac{u_{\rho}}{\rho}\left(N-2+a+(1-a) g\left(u_{\rho}^{2} a\right)\right) \leq 0,
$$

for $\rho \in(1, R(a))$. Since

$$
\begin{gathered}
F(u)=\frac{-u_{\rho}}{\rho(\rho+1) g\left(\left(u_{\rho}\right)^{2}\right)}\left[\rho(N-1)\left(1-a+a g\left(\left(u_{\rho}\right)^{2} a\right)\right)-\right. \\
\left.(\rho+1) g\left(\left(u_{\rho}\right)^{2}\right)\left(N-2+a+(1-a) g\left(\left(u_{\rho}\right)^{2} a\right)\right)\right],
\end{gathered}
$$

setting

$$
\begin{gathered}
k(\rho)= \\
\rho(N-1)\left(1-a+a g\left(\left(u_{\rho}\right)^{2} a\right)\right)-(\rho+1) g\left(\left(u_{\rho}\right)^{2}\right)\left(N-2+a+(1-a) g\left(\left(u_{\rho}\right)^{2} a\right)\right),
\end{gathered}
$$
we have that $F(u) \leq 0$ if and only if $k(\rho) \leq 0$. From

$$
\frac{d}{d \rho} g\left(\left(u_{\rho}\right)^{2}\right) \leq \frac{d}{d \rho} g\left(\left(u_{\rho}\right)^{2} a\right) \leq 0,
$$

and $N-2+a \geq(N-1) a$ for $N \geq 2$, applying Lemma [5] we have that

$$
\begin{gathered}
k^{\prime}(\rho)=(N-1)\left(1-a+a g\left(\left(u_{\rho}\right)^{2} a\right)-g\left(\left(u_{\rho}\right)^{2}\right)\left(N-2+a+(1-a) g\left(\left(u_{\rho}\right)^{2} a\right)\right)+\right. \\
\rho(N-1) a \frac{d}{d \rho} g\left(\left(u_{\rho}\right)^{2} a\right)-(\rho+1) \frac{d}{d \rho} g\left(\left(u_{\rho}\right)^{2}\right)\left(N-2+a+(1-a) g\left(\left(u_{\rho}\right)^{2} a\right)\right)- \\
(\rho+1) g\left(\left(u_{\rho}\right)^{2}\right)(1-a) \frac{d}{d \rho} g\left(\left(u_{\rho}\right)^{2} a\right) \geq \\
\rho\left[(N-1) a \frac{d}{d \rho} g\left(\left(u_{\rho}\right)^{2} a\right)-\frac{d}{d \rho} g\left(\left(u_{\rho}\right)^{2}\right)\left(N-2+a+(1-a) g\left(\left(u_{\rho}\right)^{2} a\right)\right)-\right. \\
\left.g\left(\left(u_{\rho}\right)^{2}\right)(1-a) \frac{d}{d \rho} g\left(\left(u_{\rho}\right)^{2} a\right)\right] \geq \\
\rho(N-1) a\left(\frac{d}{d \rho} g\left(\left(u_{\rho}\right)^{2} a\right)-\frac{d}{d \rho} g\left(\left(u_{\rho}\right)^{2}\right)\right) \geq 0 .
\end{gathered}
$$

Since the function $k(\rho)$ is non decreasing, it follows that $F(u) \leq 0$, for every $\rho \in(1, R(a))$, if and only if

We have that

$$
k(R(a)) \leq 0
$$

$$
\begin{gathered}
k(R(a))=R(a)(N-1)\left(1-a+a g\left(\left(u_{\rho}(R(a))\right)^{2} a\right)\right)- \\
(R(a)+1) g\left(\left(u_{\rho}(R(a))\right)^{2}\right)\left(N-2+a+(1-a) g\left(\left(u_{\rho}(R(a))\right)^{2} a\right)\right) \leq \\
(N-1)\left[R(a)(1-a)-g\left(\left(u_{\rho}(R(a))\right)^{2}\right) a\right] \leq \\
(N-1)\left[R(1)(1-a)-g\left(\left(u_{\rho}(R(a))\right)^{2}\right) a\right] \leq \\
(N-1)\left[R(1)(1-a)-\frac{g\left(\left(u_{\rho}(R(a))\right)^{2}\right)}{2}\right] .
\end{gathered}
$$

We define $R(a)$ to be a solution to

$$
R(1)(1-a)-\frac{g\left(\left(u_{\rho}(R(a))\right)^{2}\right)}{2}=0 .
$$


d) In order to solve (10) for the unknown $R(a)$, recalling that $1-a=\cos ^{2} \theta_{N-1}=$ $c^{2}$, let

$$
h(r)=\sqrt{\frac{g\left(\left(u_{\rho}(r)\right)^{2}\right)}{2 R(1)}} .
$$

The function $h$ is decreasing, differentiable and with inverse differentiable. We have that $|c|=h\left(R\left(1-c^{2}\right)\right)$, so that $R\left(1-c^{2}\right)=h^{-1}(|c|), R\left(1-c^{2}\right)$ is increasing in $|c|$ and

$$
\lim _{c \rightarrow 0} R\left(1-c^{2}\right)=R(1) .
$$

Let $0<|\bar{c}|<1 / 2$ be such that $R\left(1-\bar{c}^{2}\right) \geq 1$, so that, for $c^{2} \leq \bar{c}^{2}$, we have $R\left(1-c^{2}\right) \geq R\left(1-\bar{c}^{2}\right) \geq 1$. We have obtained that, for every $1 \geq a \geq 1-|c|^{2}$, there exists $R(a)$ such that (10) holds. It follows that

$$
k(R(a)) \leq\left[R(1)(1-a)-\frac{g\left(\left(u_{\rho}(R(a))\right)^{2}\right)}{2}\right]=0,
$$

so that the function $u$ solves $F(u) \leq 0$ for every $\rho \in(1, R(a))$.

e) Set $\Omega=\left\{\left(x_{1}, \ldots, x_{N}\right) \in \mathbb{R}^{N}: \rho \in\left(1, R\left(1-c^{2}\right)\right)\right.$ and $\left.|c|<|\bar{c}|\right\} . \Omega \subset \mathbb{R}^{N}$ is a connected, open and bounded set and $u \in W^{1,2}(\Omega) \cap C(\bar{\Omega})$ is a weak solution to $F(u) \leq 0$. The point $z=(R, 0, \ldots, 0) \in \partial \Omega$ is such that $u(z)<u(x)$, for all $x \in \Omega$. We wish to show that $\Omega$ is regular in a neighborhood of $z=(R, 0, \ldots, 0)$. Since $\frac{d}{d c} R\left(1-c^{2}\right)$ exists, in $(0,|\bar{c}|)$, to prove our claim it is sufficient to show that

$$
\lim _{c \rightarrow 0} \frac{d}{d c} R\left(1-c^{2}\right)=0 .
$$

Recalling (9), we have that

$$
\begin{gathered}
\frac{d}{d c} R\left(1-c^{2}\right)=\left(h^{-1}(c)\right)^{\prime}=\frac{1}{h^{\prime}\left(R\left(1-c^{2}\right)\right)}= \\
-\frac{\sqrt{2} R(1)}{N-1}\left(R\left(1-c^{2}\right)+1\right) \frac{\left(g\left(\left(u_{\rho}\left(R\left(1-c^{2}\right)\right)\right)^{2}\right)\right)^{3 / 2}}{\left(u_{\rho}\left(R\left(1-c^{2}\right)\right)^{2} g^{\prime}\left(\left(u_{\rho}\left(R\left(1-c^{2}\right)\right)\right)^{2}\right)\right.} .
\end{gathered}
$$

Since

$$
\lim _{c \rightarrow 0} u_{\rho}\left(R\left(1-c^{2}\right)\right)=0
$$

and

$$
\lim _{t \rightarrow 0^{+}} \frac{(g(t))^{3 / 2}}{t g^{\prime}(t)}=0
$$

it follows that

$$
\lim _{c \rightarrow 0} \frac{d}{d c} R\left(1-c^{2}\right)=0 .
$$

Since $\left.\frac{d}{d \theta} \cos \theta_{N-1}\right|_{\mid \theta_{N-1}=\frac{\pi}{2}}=1$, this shows that $\frac{d}{d \theta} R\left(1-\cos ^{2} \theta_{N-1}\right)=0$, and $\Omega$ is regular. 
f) To prove the validity of the interior ball condition at $z=(R, 0, \ldots, 0)$, it is enough to show that the second derivative of $R\left(1-c^{2}\right)$ is bounded at $c=0$, i.e. that

is bounded. Set

$$
\left|\frac{1}{c} \frac{d}{d c} R\left(1-c^{2}\right)\right|
$$

$$
t(c)=\left(u_{\rho}\left(R\left(1-c^{2}\right)\right)\right)^{2},
$$

from (111) we obtain

$$
\frac{d}{d c} R\left(1-c^{2}\right)=-\frac{\sqrt{2} R(1)}{N-1}\left(R\left(1-c^{2}\right)+1\right) \frac{(g(t(c)))^{3 / 2}}{t(c) g^{\prime}(t(c))},
$$

and from (9)

$$
\begin{gathered}
\frac{d t(c)}{d c}=2 u_{\rho}\left(R\left(1-c^{2}\right)\right) u_{\rho \rho}\left(R\left(1-c^{2}\right)\right) \frac{d}{d c}\left(R\left(1-c^{2}\right)\right)= \\
2 \sqrt{2} R(1) \frac{(g(t(c)))^{1 / 2}}{g^{\prime}(t(c))}
\end{gathered}
$$

and

$$
\frac{d}{d c}(g(t(c)))^{1 / 2}=\frac{g^{\prime}(t(c))}{2(g(t(c)))^{1 / 2}} \frac{d t(c)}{d c}=\sqrt{2} R(1) .
$$

From $g(t(0))=0$, we obtain that

$$
(g(t(c)))^{1 / 2}=\sqrt{2} R(1) c
$$

and

$$
\frac{(g(t(c)))^{3 / 2}}{c t(c) g^{\prime}(t(c))}=\sqrt{2} R(1) \frac{g(t(c))}{t(c) g^{\prime}(t(c))} .
$$

From condition (6) we obtain

$$
\left|\frac{1}{c} \frac{d}{d c} R\left(1-c^{2}\right)\right| \leq M
$$

\section{A sufficient condition for the validity of the Strong Maximum}

\section{PRINCIPLE}

Consider the case

$$
G(\xi)=\int_{0}^{\xi} \frac{g\left(\zeta^{2} / N\right)}{\zeta} d \zeta<+\infty .
$$

We wish to prove the following theorem.

Theorem 3 (The Strong Maximum Principle). Let $\Omega \subset \mathbb{R}^{N}$ be a connected, open and bounded set. Let $u \in W^{1,2}(\Omega) \cap C(\bar{\Omega})$ be a weak supersolution to

$$
\sum_{i=1}^{N-1} u_{x_{i} x_{i}}+g\left(u_{x_{N}}^{2}\right) u_{x_{N} x_{N}}=0
$$

where $g$ satisfies assumptions $(L)$ and $(G)$ and, on $(0, \bar{t}](\bar{t}$ defined in assumptions $(L))$,

$$
g^{\prime}(t) \geq 0 \quad \text { and } \quad g(t)+g^{\prime}(t) t \leq 1 .
$$




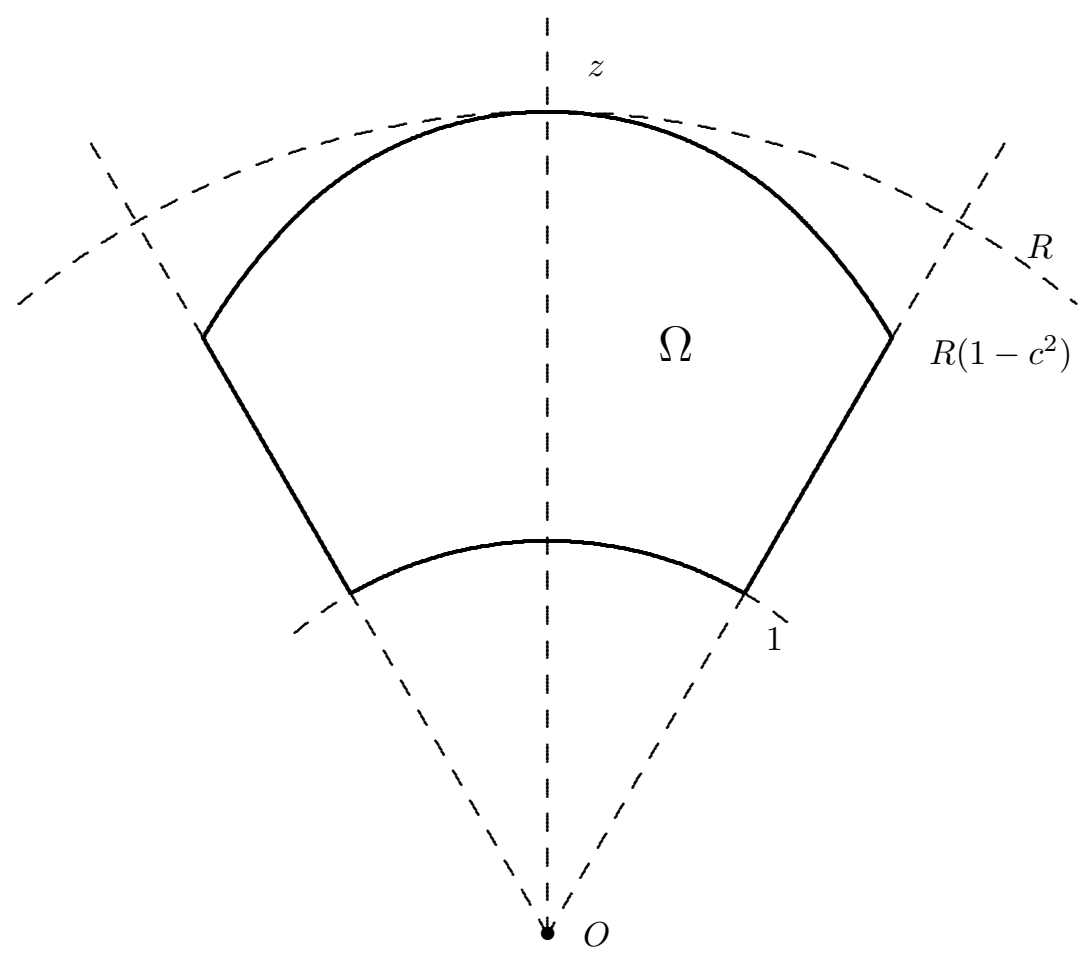

Figure $1 . \Omega$ in the case $N=2$.

Moreover, suppose that there exists $K>0$ such that, for every $0<\xi^{2} / N \leq \bar{t}$, we have

$$
\sqrt{g\left(\xi^{2} / N\right)} \leq K\left(e^{G(\xi)}-1\right) .
$$

Then, if $u$ attains its minimum in $\Omega$, it is a constant.

Remark 2. When the function g satisfies the condition

$$
g^{\prime}(t) t \leq 2 K(g(t))^{3 / 2},
$$

for every $0<t \leq \bar{t}$, then it satisfies

$$
g(t)+g^{\prime}(t) t \leq 1
$$

and

$$
\sqrt{g\left(\xi^{2} / N\right)} \leq K\left(e^{G(\xi)}-1\right)
$$

for every $0<\xi^{2} / N \leq \bar{t}$.

Indeed, since $G(\xi)<+\infty$, we have that $\lim _{t \rightarrow 0} g(t)=0$. Hence, we can suppose that $g(t) \leq 1 /(2 K+1)$, for $0<t \leq \bar{t}$, so that

$$
g(t)+g^{\prime}(t) t \leq g(t)+2 K(g(t))^{3 / 2} \leq 1
$$


Moreover, since $g(0)=0, G(0)=0$ and

$$
\left(\sqrt{g\left(\xi^{2} / N\right)}\right)^{\prime} \leq 2 K \frac{g\left(\xi^{2} / N\right)}{\xi} \leq K\left(e^{G(\xi)}-1\right)^{\prime}
$$

we obtain that

$$
\sqrt{g\left(\xi^{2} / N\right)} \leq K\left(e^{G(\xi)}-1\right) .
$$

Remark 3. Among the functions $g$ such that

$$
\lim _{t \rightarrow 0^{+}} \frac{(g(t))^{3 / 2}}{t g^{\prime}(t)}
$$

exists, there exists $K>0$ such that, for every $0<\xi^{2} / N \leq \bar{t}$, we have

$$
\sqrt{g\left(\xi^{2} / N\right)} \leq K\left(e^{G(\xi)}-1\right)
$$

if and only if

$$
\lim _{t \rightarrow 0^{+}} \frac{(g(t))^{3 / 2}}{t g^{\prime}(t)}>0
$$

An example of a map satisfying the assumptions of the theorem above, is given by

$$
g(t)=\frac{1}{(\ln (t))^{2}},
$$

for $0 \leq t \leq 1 / e^{4}$. For $0 \leq \xi^{2} / N \leq 1 / e^{4}$, we have

$$
G(\xi)=\int_{0}^{\xi} \frac{1}{\zeta\left(\ln \left(\zeta^{2} / N\right)\right)^{2}} d \zeta=-\frac{1}{2 \ln \left(\xi^{2} / N\right)},
$$

and

$$
\sqrt{g\left(\xi^{2} / N\right)}=\frac{1}{\left|\ln \left(\xi^{2} / N\right)\right|} \leq 2\left(e^{\frac{1}{2\left|\ln \left(\xi^{2} / N\right)\right|}}-1\right)=e^{G(\xi)}-1
$$

Set

$$
\mathcal{R}\left(\lambda, \lambda_{N}\right)=\left\{\left(x_{1}, \ldots, x_{N}\right):\left|x_{i}\right| \leq \lambda, \text { for } i=1, \ldots, N-1,\left|x_{N}\right| \leq \lambda_{N}\right\} .
$$

To the opposite of the proof of Lemma 4 and Theorem 1 we will build a subsolution that is not radially symmetric. This construction is provided by next theorem.

Theorem 4. Under the same assumptions on $g$ as on Theorem 3 for every $r>0$ and every $\epsilon$, there exist: $l, l_{N}$; an open convex region $\mathcal{A} \subset \mathcal{R}\left(l, l_{N}\right)$; a function $v \in W^{1,2}(\omega) \cap C^{1}(\omega) \cap C(\bar{\omega})$, where $\omega=B(\mathcal{A}, r) \backslash \overline{\mathcal{A}}$, such that

i) $0 \leq l \leq 2 K r$, and $0 \leq l_{N} \leq r / 4$;

ii)

$$
\begin{cases}v \text { is a weak solution to } F(v) \geq 0 & \text { in } \omega \\ v>0 & \text { in } \omega \\ v=0 & \text { in } \partial B(\mathcal{A}, r) \\ v \leq \epsilon & \text { in } \partial \mathcal{A} .\end{cases}
$$

Proof. Fix $r$; we can assume that $\epsilon$ is such that $0<\epsilon^{2} / r^{2} \leq \bar{t}$ and that

$$
2 \sqrt{g\left(\frac{\epsilon^{2}}{r^{2}}\right)}+\frac{1}{2} \sqrt{g\left(\frac{\epsilon^{2}}{r^{2}}\right)}\left|\ln g\left(\frac{\epsilon^{2}}{r^{2}}\right)\right| \leq \frac{1}{4 K} .
$$


Fix the origin $O^{0}=(0, \ldots, 0)$, and set polar coordinates as

$$
\left\{\begin{array}{l}
x_{1}=\rho \cos \theta_{N-1} \ldots \cos \theta_{2} \cos \theta_{1} \\
x_{2}=\rho \cos \theta_{N-1} \ldots \cos \theta_{2} \sin \theta_{1} \\
\ldots \\
x_{N}=\rho \sin \theta_{N-1} .
\end{array}\right.
$$

1) When $w$ is a radial function, setting $a=\sin ^{2} \theta_{1}, F$ reduces to

$$
F(w)=w_{\rho \rho}\left(1-a+a g\left(w_{\rho}^{2} a\right)\right)+\frac{w_{\rho}}{\rho}\left(N-2+a+(1-a) g\left(w_{\rho}^{2} a\right)\right) .
$$

For $a=1$, we seek a solution to

$$
(N-1) \frac{w_{\rho}}{\rho}+w_{\rho \rho} g\left(w_{\rho}^{2}\right)=0 .
$$

such that $w_{\rho}(R(1))=-\epsilon / r$ and $w_{\rho}(\rho)<0$, for every $\rho \in[R(1), R(1)+r)$. Consider the Cauchy problem

$$
\left\{\begin{array}{l}
\zeta^{\prime}=-\frac{N-1}{\rho} \frac{\zeta}{g\left(\zeta^{2}\right)} \\
\zeta(R(1))=-\frac{\epsilon}{r} .
\end{array}\right.
$$

We are interested in a negative solution $\zeta$. Define $R(1)$ to be the unique positive real solution to

$$
G(-\epsilon / r)-(N-1) \ln \left(\frac{R(1)+r}{R(1)}\right)=0,
$$

i.e.

$$
R(1)=\frac{r}{e^{\frac{G(-\epsilon / r)}{N-1}}-1} .
$$

Consider the unique solution $\zeta$ of (14), such that $\zeta(R(1))=-\epsilon / r$, i.e., such that

$$
G(\zeta(\rho))-G(-\epsilon / r)=\int_{\zeta(R(1))}^{\zeta(\rho)} \frac{g\left(t^{2}\right)}{t} d t=\int_{R(1)}^{\rho}-\frac{N-1}{s} d s=-(N-1) \ln \left(\frac{\rho}{R(1)}\right) .
$$

Then, for every $\rho \in[R(1), R(1)+r), G(\zeta(\rho))>0$ and $\zeta(\rho)<0$, while $\zeta(R(1)+r)=$ 0 . Setting $w_{\rho}(\rho)=\zeta(\rho)$ and

$$
w(\rho)=\int_{R(1)+r}^{\rho} w_{\rho}(s) d s
$$

we obtain that $w$ solves (13) and, for every $\rho \in(R(1), R(1)+r)$,

$$
-\epsilon / r=w_{\rho}(R(1))<w_{\rho}(\rho)<w_{\rho}(R(1)+r)=0
$$

and

$$
0=w(R(1)+r)<w(\rho)<w(R(1)) \leq \epsilon .
$$

2) Applying Lemma 5 we infer that the function $w$ defined in 1 ) is actually a solution to

$$
F(w)=w_{\rho \rho}(1-a)+\frac{w_{\rho}}{\rho}(N-2+a)+g\left(w_{\rho}^{2} a\right)\left(w_{\rho \rho} a+\frac{w_{\rho}}{\rho}(1-a)\right) \geq 0,
$$

for every $0 \leq a \leq 1$ and every $\rho \in(R(1), R(1)+r)$. 
3) Let $\bar{a}<1$. We wish to find the smallest $R(\bar{a})>0$ such that, setting

$$
w^{\bar{a}}(\rho)=w(\rho-R(\bar{a})+R(1)),
$$

the function $w^{\bar{a}}$ is a solution to

(15) $F\left(w^{\bar{a}}\right)=w_{\rho \rho}^{\bar{a}}(1-\bar{a})+\frac{w_{\rho}^{\bar{a}}}{\rho}(N-2+\bar{a})+g\left(\left(w_{\rho}^{\bar{a}}\right)^{2} \bar{a}\right)\left(w_{\rho \rho}^{\bar{a}} \bar{a}+\frac{w_{\rho}^{\bar{a}}}{\rho}(1-\bar{a})\right) \geq 0$,

for every $\rho \in(R(\bar{a}), R(\bar{a})+r)$.

Since, for the function $w$, we have

$$
(N-1) \frac{w_{\rho}(\rho)}{\rho}+g\left(w_{\rho}^{2}(\rho)\right) w_{\rho \rho}(\rho)=0,
$$

at $\rho-R(\bar{a})+R(1)$ we obtain

$$
(N-1) \frac{w_{\rho}^{\bar{a}}(\rho)}{\rho-R(\bar{a})+R(1)}+g\left(\left(w_{\rho}^{\bar{a}}(\rho)\right)^{2}\right) w_{\rho \rho}^{\bar{a}}(\rho)=0 .
$$

This equality yields

$$
\begin{gathered}
(N-2+\bar{a}) \frac{w_{\rho}^{\bar{a}}}{\rho}+\bar{a} g\left(\left(w_{\rho}^{\bar{a}}\right)^{2} \bar{a}\right) w_{\rho \rho}^{\bar{a}}= \\
\frac{w_{\rho}^{\bar{a}}}{\rho(\rho-R(\bar{a})+R(1))}\left((N+2-\bar{a})(\rho-R(\bar{a})+R(1))-\bar{a} \rho(N-1) \frac{g\left(\left(w_{\rho}^{\bar{a}}\right)^{2} \bar{a}\right)}{g\left(\left(w_{\rho}^{\bar{a}}\right)^{2}\right)}\right)
\end{gathered}
$$

and

$$
\begin{gathered}
(1-\bar{a})\left(w_{\rho \rho}^{\bar{a}}+\frac{w_{\rho}^{\bar{a}}}{\rho} g\left(\left(w_{\rho}^{\bar{a}}\right)^{2} \bar{a}\right)\right)= \\
\frac{(1-\bar{a}) w_{\rho}^{\bar{a}}}{\rho(\rho-R(\bar{a})+R(1))}\left((\rho-R(\bar{a})+R(1)) g\left(\left(w_{\rho}^{\bar{a}}\right)^{2} \bar{a}\right)-\frac{(N-1) \rho}{g\left(\left(w_{\rho}^{\bar{a}}\right)^{2}\right)}\right) .
\end{gathered}
$$

Since

$$
F\left(w^{\bar{a}}\right)=(N-2+\bar{a}) \frac{w_{\rho}^{\bar{a}}}{\rho}+\bar{a} g\left(\left(w_{\rho}^{\bar{a}}\right)^{2} \bar{a}\right) w_{\rho \rho}^{\bar{a}}+(1-\bar{a})\left(w_{\rho \rho}^{\bar{a}}+\frac{w_{\rho}^{\bar{a}}}{\rho} g\left(\left(w_{\rho}^{\bar{a}}\right)^{2}\right)\right),
$$

we obtain that $F\left(w^{\bar{a}}\right) \geq 0$ if and only if

$$
\begin{gathered}
(\rho-R(\bar{a})+R(1))\left(N-2+\bar{a}+(1-\bar{a}) g\left(\left(w_{\rho}^{\bar{a}}\right)^{2} \bar{a}\right)\right)- \\
\frac{(N-1) \rho}{g\left(\left(w_{\rho}^{\bar{a}}\right)^{2}\right)}\left(1-\bar{a}+\bar{a} g\left(\left(w_{\rho}^{\bar{a}}\right)^{2} \bar{a}\right)\right) \leq 0 .
\end{gathered}
$$

Set

Since

$$
\begin{gathered}
k(\rho)=(\rho-R(\bar{a})+R(1))\left(N-2+\bar{a}+(1-\bar{a}) g\left(\left(w_{\rho}^{\bar{a}}\right)^{2} \bar{a}\right)\right)- \\
\frac{(N-1) \rho}{g\left(\left(w_{\rho}^{\bar{a}}\right)^{2}\right)}\left(1-\bar{a}+\bar{a} g\left(\left(w_{\rho}^{\bar{a}}\right)^{2} \bar{a}\right)\right)
\end{gathered}
$$

applying Lemma [5] we have that

$$
\frac{d}{d \rho} g\left(\left(w_{\rho}^{\bar{a}}\right)^{2}\right) \leq \frac{d}{d \rho} g\left(\left(w_{\rho}^{\bar{a}}\right)^{2} \bar{a}\right) \leq 0,
$$

$$
\begin{gathered}
k^{\prime}(\rho)= \\
\left(N-2+\bar{a}+(1-\bar{a}) g\left(\left(w_{\rho}^{\bar{a}}\right)^{2} \bar{a}\right)\right)+(\rho-R(\bar{a})+R(1))(1-\bar{a}) \frac{d}{d \rho} g\left(\left(w_{\rho}^{\bar{a}}\right)^{2} \bar{a}\right)- \\
(N-1)\left(\frac{1}{g\left(\left(w_{\rho}^{\bar{a}}\right)^{2}\right)}-\frac{\rho}{\left(g\left(\left(w_{\rho}^{\bar{a}}\right)^{2}\right)\right)^{2}} \frac{d}{d \rho} g\left(\left(w_{\rho}^{\bar{a}}\right)^{2}\right)\right)\left(1-\bar{a}+\bar{a} g\left(\left(w_{\rho}^{\bar{a}}\right)^{2} \bar{a}\right)\right)-
\end{gathered}
$$




$$
\begin{gathered}
(N-1) \frac{\rho}{g\left(\left(w_{\rho}^{\bar{a}}\right)^{2}\right)} \frac{d}{d \rho} g\left(\left(w_{\rho}^{\bar{a}}(\rho)\right)^{2} \bar{a}\right) \bar{a} \leq \\
\frac{(N-1) \rho}{\left(g\left(\left(w_{\rho}^{\bar{a}}\right)^{2}\right)\right)^{2}} \frac{d}{d \rho} g\left(\left(w_{\rho}^{\bar{a}}\right)^{2}\right)\left(1-\bar{a}+\bar{a} g\left(\left(w_{\rho}^{\bar{a}}\right)^{2} \bar{a}\right)\right)-\frac{(N-1) \rho}{g\left(\left(w_{\rho}^{\bar{a}}\right)^{2}\right)} \frac{d}{d \rho} g\left(\left(w_{\rho}^{\bar{a}}(\rho)\right)^{2} \bar{a}\right) \bar{a} \leq \\
\frac{(N-1) \rho}{\left(g\left(\left(w_{\rho}^{\bar{a}}\right)^{2}\right)\right)^{2}} \frac{d}{d \rho} g\left(\left(w_{\rho}^{\bar{a}}\right)^{2}\right)\left(1-\bar{a}+\bar{a} g\left(\left(w_{\rho}^{\bar{a}}\right)^{2} \bar{a}\right)-\bar{a} g\left(\left(w_{\rho}^{\bar{a}}\right)^{2}\right)\right) \leq \\
\frac{(N-1) \rho}{\left(g\left(\left(w_{\rho}^{\bar{a}}\right)^{2}\right)\right)^{2}}(1-\bar{a}) g\left(\left(w_{\rho}^{\bar{a}}\right)^{2}\right) \frac{d}{d \rho} g\left(\left(w_{\rho}^{\bar{a}}\right)^{2}\right) \leq 0 .
\end{gathered}
$$

Since the function $k(\rho)$ is non increasing, it follows that $F\left(w^{\bar{a}}\right) \geq 0$, for every $\rho \in(R(\bar{a}), R(\bar{a})+r)$, if and only if

$$
\begin{gathered}
R(1)\left(N-2+\bar{a}+(1-\bar{a}) g\left(\left(w_{\rho}^{\bar{a}}(R(\bar{a}))\right)^{2} \bar{a}\right)\right)- \\
\frac{(N-1) R(\bar{a})}{g\left(\left(w_{\rho}^{\bar{a}}(R(\bar{a}))^{2}\right)\right.}\left(1-\bar{a}+\bar{a} g\left(\left(w_{\rho}^{\bar{a}}(R(\bar{a}))\right)^{2} \bar{a}\right)\right)= \\
R(1)\left(N-2+\bar{a}+(1-\bar{a}) g\left(\frac{\epsilon^{2}}{r^{2}} \bar{a}\right)\right)-\frac{(N-1) R(\bar{a})}{g\left(\frac{\epsilon^{2}}{r^{2}}\right)}\left(1-\bar{a}+\bar{a} g\left(\frac{\epsilon^{2}}{r^{2}} \bar{a}\right)\right) \leq 0,
\end{gathered}
$$

i.e. if and only if

$$
R(\bar{a}) \geq g\left(\frac{\epsilon^{2}}{r^{2}}\right) \frac{R(1)}{N-1} \frac{N-2+\bar{a}+g\left(\frac{\epsilon^{2}}{r^{2}} \bar{a}\right)(1-\bar{a})}{1-\bar{a}+g\left(\frac{\epsilon^{2}}{r^{2}} \bar{a}\right) \bar{a}} .
$$

Hence, we define

$$
R(\bar{a})=g\left(\frac{\epsilon^{2}}{r^{2}}\right) \frac{R(1)}{N-1} \frac{N-2+\bar{a}+g\left(\frac{\epsilon^{2}}{r^{2}} \bar{a}\right)(1-\bar{a})}{1-\bar{a}+g\left(\frac{\epsilon^{2}}{r^{2}} \bar{a}\right) \bar{a}} .
$$

4) The function $w^{\bar{a}}$ defined in point 3 ) is a solution to

$$
F\left(w^{\bar{a}}\right)=w_{\rho \rho}^{\bar{a}}(1-a)+\frac{w_{\rho}^{\bar{a}}}{\rho}(N-2+a)+g\left(\left(w_{\rho}^{\bar{a}}\right)^{2} a\right)\left(w_{\rho \rho}^{\bar{a}} a+\frac{w_{\rho}^{\bar{a}}}{\rho}(1-a)\right) \geq 0,
$$

for every $a<\bar{a}$. Indeed, applying Lemma 5 we obtain that, for every $\rho \in$ $(R(\bar{a}), R(\bar{a})+r)$,

$$
\begin{gathered}
(\rho-R(\bar{a})+R(1))\left(N-2+a+(1-a) g\left(\left(w_{\rho}^{\bar{a}}\right)^{2} a\right)\right)- \\
\frac{(N-1) \rho}{g\left(\left(w_{\rho}^{\bar{a}}\right)^{2}\right)}\left(1-a+a g\left(\left(w_{\rho}^{\bar{a}}\right)^{2} a\right)\right) \leq \\
(\rho-R(\bar{a})+R(1))\left(N-2+\bar{a}+(1-\bar{a}) g\left(\left(w_{\rho}^{\bar{a}}\right)^{2} \bar{a}\right)\right)- \\
\frac{(N-1) \rho}{g\left(\left(w_{\rho}^{\bar{a}}\right)^{2}\right)}\left(1-\bar{a}+\bar{a} g\left(\left(w_{\rho}^{\bar{a}}\right)^{2} \bar{a}\right)\right) \leq 0 .
\end{gathered}
$$

5) Assume we have a partition $\alpha$ of $[0, \pi / 2], \alpha=\left\{0=\alpha_{n}<\cdots<\alpha_{1}<\right.$ $\left.\alpha_{0}=\pi / 2\right\}$. This partition defines two partitions of [0,1], given by $c_{i}=\cos \alpha_{i}$ and $s_{i}=\sin \alpha_{i}$. 
Consider the sums

$$
S_{1}(\alpha)=\sum_{i=1}^{n}\left(R\left(1-c_{i-1}^{2}\right)-R\left(1-c_{i}^{2}\right)\right) c_{i}=R(1) c_{1}+\sum_{i=1}^{n-1} R\left(1-c_{i}^{2}\right)\left(c_{i+1}-c_{i}\right)
$$

and

$$
S_{2}(\alpha)=\sum_{i=1}^{n} R\left(s_{i-1}^{2}\right)\left(s_{i-1}-s_{i}\right)=R(1)\left(1-s_{1}\right)+\sum_{i=1}^{n-1} R\left(s_{i}^{2}\right)\left(s_{i}-s_{i+1}\right),
$$

where, in the previous equalities, we have taken into account that $R\left(1-c_{n}^{2}\right)=$ $R(0)=0$. Our purpose is to provide a partition $\alpha$ and corresponding estimates for $S_{1}(\alpha)$ and $S_{2}(\alpha)$ that are independent of $\epsilon$.

The sums

$$
\sum_{i=1}^{n-1} R\left(1-c_{i}^{2}\right)\left(c_{i+1}-c_{i}\right) \quad \text { and } \quad \sum_{i=1}^{n-1} R\left(s_{i}^{2}\right)\left(s_{i}-s_{i+1}\right)
$$

are Riemann sums for the integrals

$$
\int_{c_{1}}^{1} R\left(1-c^{2}\right) d c \quad \text { and } \quad \int_{0}^{s_{1}} R\left(s^{2}\right) d s
$$

Consider the first integral. From

$$
\frac{N-1-c^{2}+g\left(\frac{\epsilon^{2}}{r^{2}}\left(1-c^{2}\right)\right) c^{2}}{c^{2}+g\left(\frac{\epsilon^{2}}{r^{2}}\left(1-c^{2}\right)\right)\left(1-c^{2}\right)} \leq \frac{N-1}{c^{2}}
$$

we obtain that

$$
\begin{gathered}
\int_{c_{1}}^{1} R\left(1-c^{2}\right) d c=g\left(\frac{\epsilon^{2}}{r^{2}}\right) \\
\frac{R(1)}{N-1} \int_{c_{1}}^{1} \frac{N-1-c^{2}+g\left(\frac{\epsilon^{2}}{r^{2}}\left(1-c^{2}\right)\right) c^{2}}{c^{2}+g\left(\frac{\epsilon^{2}}{r^{2}}\left(1-c^{2}\right)\right)\left(1-c^{2}\right)} d c \leq \\
R(1) g\left(\frac{\epsilon^{2}}{r^{2}}\right) \int_{c_{1}}^{1} \frac{d c}{c^{2}} .
\end{gathered}
$$

Set

$$
\begin{gathered}
S_{x}^{*}(c)=R(1) c+R(1) g\left(\frac{\epsilon^{2}}{r^{2}}\right) \int_{c}^{1} \frac{d b}{b^{2}}=R(1)\left(c+g\left(\frac{\epsilon^{2}}{r^{2}}\right)\left(\frac{1}{c}-1\right)\right)= \\
R(1) g\left(\frac{\epsilon^{2}}{r^{2}}\right)\left(\frac{c}{g\left(\frac{\epsilon^{2}}{r^{2}}\right)}+\frac{1}{c}-1\right) .
\end{gathered}
$$

Evaluating the last term at the minimum point $c=\sqrt{g\left(\frac{\epsilon^{2}}{r^{2}}\right)}$, we obtain

$$
\begin{gathered}
S_{x}^{*}\left(\sqrt{g\left(\frac{\epsilon^{2}}{r^{2}}\right)}\right)=R(1) g\left(\frac{\epsilon^{2}}{r^{2}}\right)\left(\frac{2}{\sqrt{g\left(\frac{\epsilon^{2}}{r^{2}}\right)}}-1\right)= \\
\frac{2 r \sqrt{g\left(\frac{\epsilon^{2}}{r^{2}}\right)}}{e^{G(\epsilon / r)}-1}-R(1) g\left(\frac{\epsilon^{2}}{r^{2}}\right) .
\end{gathered}
$$

We fix $c_{1}=\sqrt{g\left(\frac{\epsilon^{2}}{r^{2}}\right)}$, so that $\alpha_{1}=\arccos \sqrt{g\left(\frac{\epsilon^{2}}{r^{2}}\right)}$. 
Consider the second integral

$$
\int_{0}^{s_{1}} R\left(s^{2}\right) d s
$$

From

$$
\frac{N-2+s^{2}+g\left(\frac{\epsilon^{2}}{r^{2}} s^{2}\right)\left(1-s^{2}\right)}{1-s^{2}+g\left(\frac{\epsilon^{2}}{r^{2}}\right) s^{2}} \leq \frac{N-2+s^{2}+g\left(\frac{\epsilon^{2}}{r^{2}} s^{2}\right)\left(1-s^{2}\right)}{1-s^{2}}
$$

we obtain that

$$
\begin{gathered}
\int_{0}^{s_{1}} R\left(s^{2}\right) d s=g\left(\frac{\epsilon^{2}}{r^{2}}\right) \frac{R(1)}{N-1} \int_{0}^{s_{1}} \frac{N-2+s^{2}+g\left(\frac{\epsilon^{2}}{r^{2}} s^{2}\right)\left(1-s^{2}\right)}{1-s^{2}+g\left(\frac{\epsilon^{2}}{r^{2}} s^{2}\right) s^{2}} d s \leq \\
g\left(\frac{\epsilon^{2}}{r^{2}}\right) \frac{R(1)}{N-1} \int_{0}^{s_{1}} \frac{N-2+s^{2}+g\left(\frac{\epsilon^{2}}{r^{2}}\right)\left(1-s^{2}\right)}{1-s^{2}} d s .
\end{gathered}
$$

Set

$$
\begin{gathered}
S_{y}^{*}(s)=R(1)(1-s)+g\left(\frac{\epsilon^{2}}{r^{2}}\right) \frac{R(1)}{N-1} \int_{0}^{s} \frac{N-2+b^{2}+g\left(\frac{\epsilon^{2}}{r^{2}}\right)\left(1-b^{2}\right)}{1-b^{2}} d b= \\
R(1)(1-s)+g\left(\frac{\epsilon^{2}}{r^{2}}\right) \frac{R(1)}{N-1}\left[\left(g\left(\frac{\epsilon^{2}}{r^{2}}\right)-1\right) s+\frac{N-1}{2} \ln \left(\frac{1+s}{1-s}\right)\right] .
\end{gathered}
$$

Since

$$
1-\sqrt{1-g\left(\frac{\epsilon^{2}}{r^{2}}\right)} \leq g\left(\frac{\epsilon^{2}}{r^{2}}\right)
$$

evaluating the last term at the point $s_{1}=\sin \alpha_{1}=\sqrt{1-g\left(\frac{\epsilon^{2}}{r^{2}}\right)}$, we obtain

$$
\begin{gathered}
S_{x}^{*}\left(\sqrt{1-g\left(\frac{\epsilon^{2}}{r^{2}}\right)}\right)=R(1)\left(1-\sqrt{1-g\left(\frac{\epsilon^{2}}{r^{2}}\right)}\right)+ \\
g\left(\frac{\epsilon^{2}}{r^{2}}\right) \frac{R(1)}{N-1}\left[\left(g\left(\frac{\epsilon^{2}}{r^{2}}\right)-1\right) \sqrt{1-g\left(\frac{\epsilon^{2}}{r^{2}}\right)}-\frac{1}{2} \ln \left(\frac{1-\sqrt{1-g\left(\frac{\epsilon^{2}}{r^{2}}\right)}}{1+\sqrt{1-g\left(\frac{\epsilon^{2}}{r^{2}}\right)}}\right)\right] \leq \\
R(1) \sqrt{g\left(\frac{\epsilon^{2}}{r^{2}}\right)}\left(\sqrt{g\left(\frac{\epsilon^{2}}{r^{2}}\right)}+\frac{1}{2} \sqrt{g\left(\frac{\epsilon^{2}}{r^{2}}\right)}\left|\ln g\left(\frac{\epsilon^{2}}{r^{2}}\right)\right|\right) .
\end{gathered}
$$

To define the other points of the required partition $\alpha$, consider the integrals

$$
\int_{c_{1}}^{1} R\left(1-c^{2}\right) d c \quad \text { and } \quad \int_{0}^{s_{1}} R\left(s^{2}\right) d s
$$

Set

$$
\sigma=R(1) g\left(\frac{\epsilon^{2}}{r^{2}}\right) \text {. }
$$

By the basic theorem of Riemann integration, taking a partition $\alpha$ with mesh size small enough, the value of the Riemann sums

$$
\sum_{i=1}^{n-1} R\left(1-c_{i}^{2}\right)\left(c_{i+1}-c_{i}\right) \quad \text { and } \quad \sum_{i=1}^{n-1} R\left(s_{i}^{2}\right)\left(s_{i}-s_{i+1}\right)
$$


differs from

$$
\int_{c_{1}}^{1} R\left(1-c^{2}\right) d c \quad \text { and } \quad \int_{0}^{s_{1}} R\left(s^{2}\right) d s
$$

by less than $\sigma$. In particular we obtain

$$
\begin{gathered}
S_{1}(\alpha)=R(1) c_{1}+\sum_{i=1}^{n-1} R\left(1-c_{i}^{2}\right)\left(c_{i+1}-c_{i}\right) \leq R(1) c_{1}+\int_{c_{1}}^{1} R\left(1-c^{2}\right) d c+\sigma \leq \\
\frac{2 r \sqrt{g\left(\frac{\epsilon^{2}}{r^{2}}\right)}}{e^{\frac{G(\epsilon / r)}{N-1}}-1} \leq 2 K r
\end{gathered}
$$

and

$$
\begin{gathered}
S_{2}(\alpha)=R(1)\left(1-s_{1}\right)+\sum_{i=1}^{n-1} R\left(s_{i}^{2}\right)\left(s_{i}-s_{i+1}\right) \leq \\
R(1)\left(1-s_{1}\right)+\int_{0}^{s_{1}} R\left(s^{2}\right) d s+\sigma \leq \\
\frac{r \sqrt{g\left(\frac{\epsilon^{2}}{r^{2}}\right)}}{e^{\frac{G(\epsilon) r)}{N-1}}-1}\left(2 \sqrt{g\left(\frac{\epsilon^{2}}{r^{2}}\right)}+\frac{1}{2} \sqrt{g\left(\frac{\epsilon^{2}}{r^{2}}\right)}\left|\ln g\left(\frac{\epsilon^{2}}{r^{2}}\right)\right|\right) \leq \frac{r}{4} .
\end{gathered}
$$

6) With respect to the coordinates fixed at the beginning of the proof, consider $x_{i} \geq 0$. Set

$$
\mathcal{D}_{0}=\left\{\left(x_{1}, \ldots, x_{N}\right): R(1)<\rho<R(1)+r, \sqrt{a_{1}} \leq \sin \theta_{N-1} \leq \sqrt{a_{0}}=1\right\}
$$

and on $\mathcal{D}_{0}$ define the function

$$
v_{0}\left(x_{1}, \ldots, x_{N}\right)=w\left(\rho\left(x_{1}, \ldots, x_{N}\right)\right) .
$$

By point 1$)$, the function $v_{0}$ is of class $C^{2}\left(\operatorname{int}\left(\mathcal{D}_{0}\right)\right)$ and satisfies, pointwise, the inequality $F\left(v_{0}\right) \geq 0$. Having defined $v_{0}$, define $v_{1}$ as follows. Set:

$$
\begin{gathered}
O_{1}\left(\theta_{1}, \ldots, \theta_{N-2}\right)=\left(O_{x_{1}}^{1}, \ldots, O_{x_{N}}^{1}\right)= \\
\left(R(1)-R\left(a_{1}\right)\right)\left(\sqrt{1-a_{1}} \cos \theta_{N-2} \ldots \cos \theta_{1}, \sqrt{1-a_{1}} \cos \theta_{N-2} \ldots \sin \theta_{1}, \ldots, \sqrt{a_{1}}\right), \\
\rho_{1}\left(x_{1}, \ldots, x_{N}\right)=\sqrt{\left(x_{1}-O_{x_{1}}^{1}\right)^{2}+\cdots+\left(x_{N}-O_{x_{N}}^{1}\right)^{2}}
\end{gathered}
$$

and

$$
\sin \theta_{N-1}^{1}\left(x_{1}, \ldots, x_{N}\right)=\frac{x_{N}-O_{x_{N}}^{1}}{\rho_{1}\left(x_{1}, \ldots, x_{N}\right)} .
$$

Recalling the definition of $w^{a_{1}}$ in 3$)$, consider

$$
\begin{gathered}
\mathcal{D}_{1}=\left\{\left(x_{1}, \ldots, x_{N}\right): R\left(a_{1}\right)<\rho_{1}\left(x_{1}, \ldots, x_{N}\right)<R\left(a_{1}\right)+r,\right. \\
\left.\sqrt{a_{2}} \leq \sin \theta_{N-1}^{1}\left(x_{1}, \ldots, x_{N}\right) \leq \sqrt{a_{1}}\right\}
\end{gathered}
$$

and, on $\mathcal{D}_{1}$, set

$$
v_{1}\left(x_{1}, \ldots, x_{N}\right)=w^{a_{1}}\left(\rho_{1}\left(x_{1}, \ldots, x_{N}\right)\right) .
$$

The function $v_{1}$ is of class $C^{2}\left(\operatorname{int}\left(\mathcal{D}_{1}\right)\right)$. We claim that $v_{1}$ still satisfies $F\left(v_{1}\right) \geq 0$. Remark that the set of the points $O^{1}$ is equal to

$$
\mathcal{O}^{1}=\left\{\left(x_{1}, \ldots, x_{N}\right): \rho=R(1)-R\left(a_{1}\right), \quad \sin \theta_{N-1}=\sqrt{a_{1}}\right\}
$$


and that for every point $p \in \mathcal{D}_{1}$, the corresponding point $O^{1}(p)$ is the projection of $p$ on $\mathcal{O}^{1}$, while $\rho_{1}(p)=d\left(p, \mathcal{O}^{1}\right)$. Then we obtain

$$
\begin{array}{cc}
\frac{\partial \rho_{1}}{\partial \theta_{i}}=0 \quad \text { for every } \quad i=1, \ldots, N-2, \\
\frac{\partial O_{x_{i}}^{1}}{\partial x_{i}} \geq 0 \quad \text { for every } \quad i=1, \ldots, N-1, \\
\frac{\partial O_{x_{N}}^{1}}{\partial x_{N}}=0 .
\end{array}
$$

and

$$
\begin{gathered}
\nabla v_{1}=\frac{w_{\rho}^{a_{1}}\left(\rho_{1}\right)}{\rho_{1}}\left(x_{1}-O_{x_{1}}^{1}, \ldots, x_{N}-O_{x_{N}}^{1}\right)= \\
w_{\rho}^{a_{1}}\left(\rho_{1}\right)\left(\cos \theta_{N-1}^{1} \ldots \cos \theta_{1}, \cos \theta_{N-1}^{1} \ldots \sin \theta_{1}, \ldots, \sin \theta_{N-1}^{1}\right), \\
\left(v_{1}\right)_{x_{i} x_{i}}=w_{\rho \rho}^{a_{1}}\left(\rho_{1}\right)\left(\frac{x_{i}-O_{x_{i}}^{1}}{\rho_{1}}\right)^{2}+\frac{w_{\rho}^{a_{1}}\left(\rho_{1}\right)}{\rho_{1}}\left(1-\left(\frac{x_{i}-O_{x_{i}}^{1}}{\rho_{1}}\right)^{2}-\frac{\partial O_{x_{i}}^{1}}{\partial x_{i}}\right)= \\
w_{\rho \rho}^{a_{1}}\left(\rho_{1}\right) \cos ^{2} \theta_{N-1} \ldots \sin ^{2} \theta_{i-1}+\frac{w_{\rho}^{a_{1}}\left(\rho_{1}\right)}{\rho_{1}}\left(1-\cos ^{2} \theta_{N-1} \ldots \sin ^{2} \theta_{i-1}-\frac{\partial O_{x_{i}}^{1}}{\partial x_{i}}\right) .
\end{gathered}
$$

Then

$$
\begin{gathered}
F\left(v_{1}\right)=\sum_{i=1}^{N-1}\left(v_{1}\right)_{x_{i} x_{i}}+\left(v_{1}\right)_{x_{N} x_{N}} g\left(\left(v_{1}\right)_{x_{N}}^{2}\right)= \\
w_{\rho \rho}^{a_{1}}\left(\rho_{1}\right)\left(\cos ^{2} \theta_{N-1}^{1}+\sin ^{2} \theta_{N-1}^{1} g\left(\left(w_{\rho}^{a_{1}}\left(\rho_{1}\right)\right)^{2} \sin ^{2} \theta_{N-1}^{1}\right)\right)+ \\
\frac{w_{\rho}^{a_{1}}\left(\rho_{1}\right)}{\rho_{1}}\left(N-2+\sin ^{2} \theta_{N-1}^{1}+\cos ^{2} \theta_{N-1}^{1} g\left(\left(w_{\rho}^{a_{1}}\left(\rho_{1}\right)\right)^{2} \sin ^{2} \theta_{N-1}^{1}\right)\right)- \\
\frac{w_{\rho}^{a_{1}}\left(\rho_{1}\right)}{\rho_{1}} \sum_{i=1}^{N-1} \frac{\partial O_{x_{i}}^{1}}{\partial x_{i}} \geq 0
\end{gathered}
$$

since $w^{a_{1}}\left(\rho_{1}\right)$ verifies equation (15).

The sets $\mathcal{D}_{0}$ and $\mathcal{D}_{1}$ intersect on $\sin \theta_{2}\left(x_{1}, \ldots, x_{N}\right)=\sin \theta_{2}^{1}\left(x_{1}, \ldots, x_{N}\right)=\sqrt{a_{1}}$. For a point $\left(x_{1}, \ldots, x_{N}\right)$ in this intersection we have

$$
\rho_{1}\left(x_{1}, \ldots, x_{N}\right)=\rho\left(x_{1}, \ldots, x_{N}\right)-\left(R(1)-R\left(a_{1}\right)\right) .
$$

Hence, on $\mathcal{D}_{0} \cap \mathcal{D}_{1}$

$$
R\left(a_{1}\right) \leq \rho_{1}\left(x_{1}, \ldots, x_{N}\right) \leq R\left(a_{1}\right)+r
$$

if and only if

$$
R(1) \leq \rho\left(x_{1}, \ldots, x_{N}\right) \leq R(1)+r,
$$

and the functions $v_{0}$ and $v_{1}$ coincide:

$$
\begin{gathered}
v_{1}\left(x_{1}, \ldots, x_{N}\right)=w^{a_{1}}\left(\rho_{1}\left(x_{1}, \ldots, x_{N}\right)\right)=w\left(\rho_{1}\left(x_{1}, \ldots, x_{N}\right)+R(1)-R\left(a_{1}\right)\right)= \\
w\left(\rho\left(x_{1}, \ldots, x_{N}\right)\right)=v_{0}\left(x_{1}, \ldots, x_{N}\right) .
\end{gathered}
$$

The formula

$$
\bar{v}\left(x_{1}, \ldots, x_{N}\right)= \begin{cases}v_{0}\left(x_{1}, \ldots, x_{N}\right) & \text { on } \mathcal{D}_{0} \\ v_{1}\left(x_{1}, \ldots, x_{N}\right) & \text { on } \mathcal{D}_{1}\end{cases}
$$

defines a function $\bar{v}$ in $C^{0}\left(\operatorname{int}\left(\mathcal{D}_{0} \cup \mathcal{D}_{1}\right)\right)$. We claim that it is also in $C^{1}\left(\operatorname{int}\left(\mathcal{D}_{0} \cup \mathcal{D}_{1}\right)\right)$. 
In fact, we have

$$
\begin{gathered}
\nabla v_{0}\left(x_{1}, \ldots, x_{N}\right)=\frac{w_{\rho}\left(\rho\left(x_{1}, \ldots, x_{N}\right)\right)}{\rho\left(x_{1}, \ldots, x_{N}\right)}\left(x_{1}, \ldots, x_{N}\right), \\
\nabla v_{1}\left(x_{1}, \ldots, x_{N}\right)=\frac{w_{\rho}^{a_{1}}\left(\rho_{1}\left(x_{1}, \ldots, x_{N}\right)\right)}{\rho_{1}\left(x_{1}, \ldots, x_{N}\right)}\left(x_{1}-O_{x_{1}}^{1}, \ldots, x_{N}-O_{x_{N}}^{1}\right)
\end{gathered}
$$

and, on $\mathcal{D}_{0} \cap \mathcal{D}_{1}$,

$$
\begin{gathered}
\frac{1}{\rho_{1}\left(x_{1}, \ldots, x_{N}\right)}\left(x_{1}-O_{x_{1}}^{1}, \ldots, x_{N}-O_{x_{N}}^{1}\right)= \\
\frac{1}{\rho\left(x_{1}, \ldots, x_{N}\right)}\left(x_{1}, \ldots, x_{N}\right) .
\end{gathered}
$$

On $\operatorname{int}\left(\mathcal{D}_{0}\right)$ and $\operatorname{int}\left(\mathcal{D}_{1}\right)$, the function $\bar{v}$ is of class $C^{2}$ and satisfies, pointwise, the inequality $F(\bar{v}) \geq 0$. We claim that $\bar{v}$ is also in $W^{1,2}\left(\operatorname{int}\left(\mathcal{D}_{0} \cup \mathcal{D}_{1}\right)\right)$ and that it is a weak solution to $F(\bar{v}) \geq 0$ on $\operatorname{int}\left(\mathcal{D}_{0} \cup \mathcal{D}_{1}\right)$. In fact, for every $\eta \in C_{0}^{\infty}\left(\operatorname{int}\left(\mathcal{D}_{0} \cup \mathcal{D}_{1}\right)\right)$, applying the divergence theorem separately to $\operatorname{int}\left(\mathcal{D}_{0}\right)$ and to $\operatorname{int}\left(\mathcal{D}_{1}\right)$, we obtain

$$
\begin{gathered}
\int_{\operatorname{int}\left(\mathcal{D}_{0} \cup \mathcal{D}_{1}\right)}\left[\operatorname{div} \nabla_{\nabla v} L(\nabla \bar{v}(x)) \eta(x)+\langle\nabla L(\nabla \bar{v}(x)), \nabla \eta(x)\rangle\right] d x= \\
\int_{\operatorname{int}\left(\mathcal{D}_{0}\right) \cup \operatorname{int}\left(\mathcal{D}_{1}\right)}\left[\operatorname{div} \nabla_{\nabla v} L(\nabla \bar{v}(x)) \eta(x)+\langle\nabla L(\nabla \bar{v}(x)), \nabla \eta(x)\rangle\right] d x= \\
\int_{\partial\left(\operatorname{int}\left(\mathcal{D}_{0}\right)\right)} \eta(x)\langle\nabla L(\nabla \bar{v}(x)), \mathbf{n}(x)\rangle d l+\int_{\partial\left(\operatorname{int}\left(\mathcal{D}_{1}\right)\right)} \eta(x)\langle\nabla L(\nabla \bar{v}(x)), \mathbf{n}(x)\rangle d l= \\
\int_{\mathcal{D}_{0} \cap\left\{\sin \theta_{N-1}=\sqrt{a_{1}}\right\}} \eta(x)\langle\nabla L(\nabla \bar{v}(x)), \mathbf{n}(x)\rangle d l+ \\
\int_{\mathcal{D}_{1} \cap\left\{\sin \theta_{N-1}^{1}=\sqrt{a_{1}}\right\}} \eta(x)\langle\nabla L(\nabla \bar{v}(x)), \mathbf{n}(x)\rangle d l .
\end{gathered}
$$

The last term equals zero, since $\bar{v} \in C^{1}\left(\operatorname{int}\left(\mathcal{D}_{0} \cup \mathcal{D}_{1}\right)\right)$. Hence, when if $\eta \geq 0$, we have that

as we wanted to show.

$$
\int_{i n t\left(\mathcal{D}_{0} \cup \mathcal{D}_{1}\right)}\langle\nabla L(\nabla \bar{v}(x)), \nabla \eta(x)\rangle d x \leq 0,
$$

Assuming defined $O^{n-2}\left(\theta_{1}, \ldots, \theta_{N-2}\right)$ and a function $v \in C^{1}\left(\operatorname{int}\left(\mathcal{D}_{0} \cup \cdots \cup\right.\right.$ $\left.\mathcal{D}_{n-2}\right)$ ), consider

$$
\begin{gathered}
O^{n-1}\left(\theta_{1}, \ldots, \theta_{N-2}\right)=\left(O_{x_{1}}^{n-1}, \ldots, O_{x_{N}}^{n-1}\right)= \\
O^{n-2}\left(\theta_{1}, \ldots, \theta_{N-2}\right)+\left(R\left(a_{n-2}\right)-R\left(a_{n-1}\right)\right) \\
\left(\sqrt{1-a_{n-1}} \cos \theta_{N-2} \ldots \cos \theta_{1}, \sqrt{1-a_{n-1}} \cos \theta_{N-2} \ldots \sin \theta_{1}, \ldots, \sqrt{a_{n-1}}\right) .
\end{gathered}
$$

Set

$$
\begin{gathered}
\rho_{n-1}\left(x_{1}, \ldots, x_{N}\right)=\sqrt{\left(x_{1}-O_{x_{1}}^{n-1}\right)^{2}+\cdots+\left(x_{N}-O_{x_{N}}^{n-1}\right)^{2}}, \\
\sin \theta_{N-1}^{n-1}\left(x_{1}, \ldots, x_{N}\right)=\frac{x_{N}-O_{x_{N}}^{n-1}}{\rho_{n-1}\left(x_{1}, \ldots, x_{N}\right)} \\
\mathcal{D}_{n-1}=\left\{\left(x_{1}, \ldots, x_{N}\right): R\left(a_{n}\right)<\rho_{n-1}\left(x_{1}, \ldots, x_{N}\right)<R\left(a_{n-1}\right)+r\right. \\
\left.0=\sqrt{a_{n}} \leq \sin \theta_{N-1}^{n-1}\left(x_{1}, \ldots, x_{N}\right) \leq \sqrt{a_{n-1}}\right\}
\end{gathered}
$$




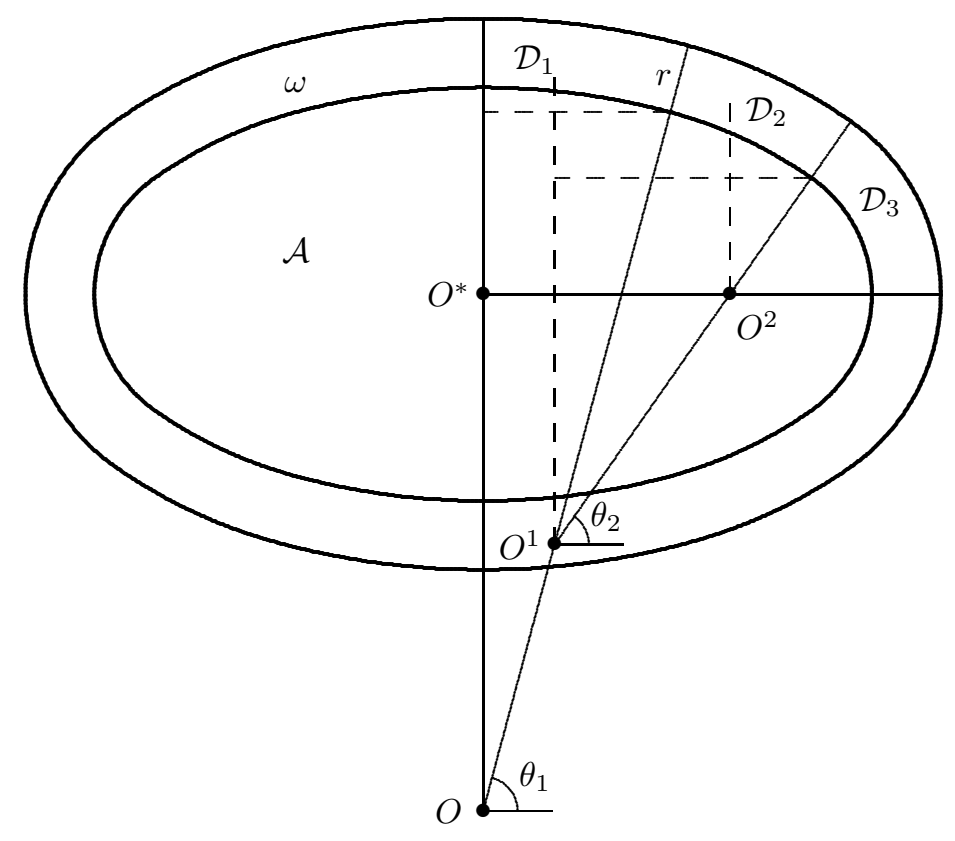

Figure 2. The sets $\omega$ and $\mathcal{A}$ in the case $N=2$ and $n=3$.

and define on $\mathcal{D}_{n-1}$ the function

$$
v_{n-1}\left(x_{1}, \ldots, x_{N}\right)=w^{a_{n-1}}\left(\rho_{n-1}\left(x_{1}, \ldots, x_{N}\right)\right) .
$$

Set $\mathcal{D}=\operatorname{int}\left(\mathcal{D}_{0} \cup \cdots \cup \mathcal{D}_{n-1}\right)$, the same considerations as before imply that the function

$$
\bar{v}\left(x_{1}, \ldots, x_{N}\right)= \begin{cases}v_{0}\left(x_{1}, \ldots, x_{N}\right) & \text { on } \mathcal{D}_{0} \\ \ldots & \ldots \\ v_{n-1}\left(x_{1}, \ldots, x_{N}\right) & \text { on } \mathcal{D}_{n-1}\end{cases}
$$

is such that $\bar{v} \in W^{1,2}(\mathcal{D}) \cap C^{1}(\mathcal{D}) \cap C(\overline{\mathcal{D}})$ and it is a weak solution to $F(\bar{v}) \geq 0$ on $\mathcal{D}$. This completes the construction of $\bar{v}$ as a weak solution to $F(\bar{v}) \geq 0$ on $\mathcal{D}_{0} \cup \cdots \cup \mathcal{D}_{N-1}$.

Set $O^{*}=\left(O_{x_{1}}^{*}, \ldots, O_{x_{N}}^{*}\right)=\left(0, \ldots, 0, R(1)-l_{N}\right)$. We have that

$$
\begin{gathered}
\mathcal{D}_{0} \cup \cdots \cup \mathcal{D}_{n-1} \subset\left\{\left(x_{1}, \ldots, x_{N}\right): 0 \leq x_{i} \leq l+r \text { for } i=1, \ldots, N-1,\right. \\
\left.\left.O_{x_{N}}^{*} \leq x_{N} \leq O_{x_{N}}^{*}+l_{N}+r\right)\right\} .
\end{gathered}
$$

Define the full domain $\omega$ and the solution by symmetry with respect to the point $O^{*}$. Figure 2 shows this construction in dimension $N=2$ and for $n-1=2$. Hence the solution will be in $W^{1,2}(\omega) \cap C^{1}(\omega) \cap C(\bar{\omega})$ and a weak solution of $F(v) \geq 0$ on $\omega$.

7) The previous construction yields a region $\mathcal{A}$ centered in $O^{*}$, a corresponding region $\omega$ and a function $v$ that solves (12). The change of coordinates $\hat{x}_{1}=$ $x_{1}, \ldots, \hat{x}_{N-1}=x_{N-1}, \hat{x}_{N}=x_{N}-O_{N}^{*}$, centers $\mathcal{A}$ at the origin and proves the 


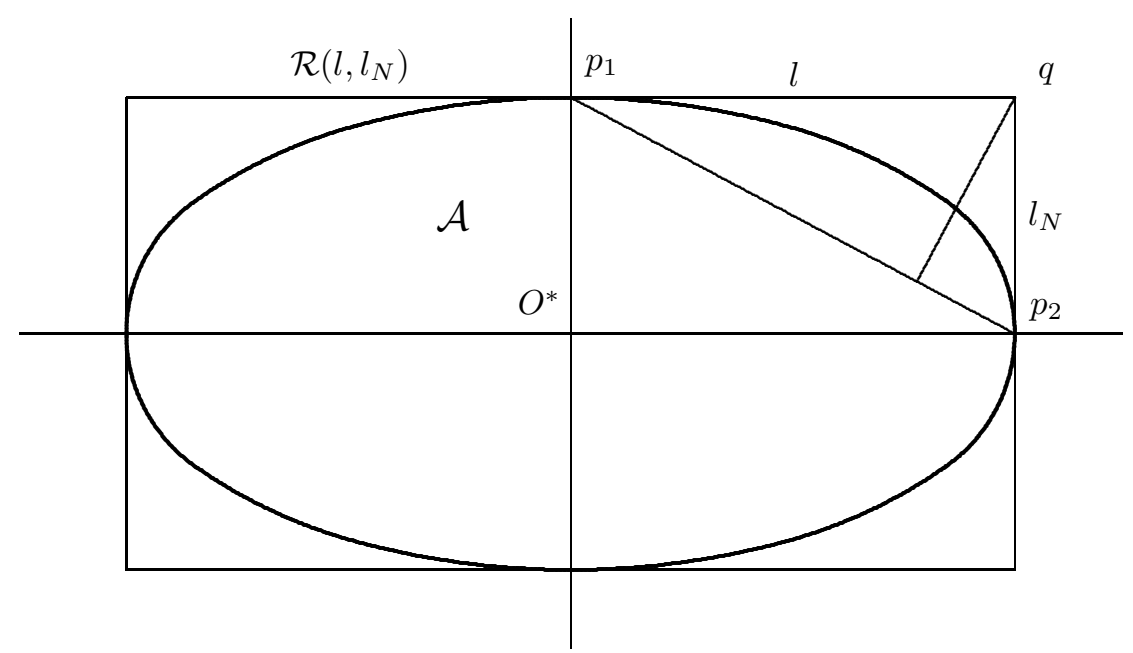

Figure 3. $\mathcal{A}$ and $\mathcal{R}\left(O^{*}, l, l_{N}\right)$ in the case $N=2$.

theorem.

In order to prove Theorem 3 we need this further lemma.

Lemma 6. Consider the sets $\mathcal{A}$ and $\mathcal{R}\left(O^{*}, l, l_{N}\right)$, where $\mathcal{A}, O^{*}, l, l_{N}$ have been defined in Theorem 3. Then, for every $p \in \partial \mathcal{R}$,

$$
d(p, \overline{\mathcal{A}})<l_{N}
$$

Proof. Set $q=O^{*}+\left(l, \ldots, l, l_{N}\right)$. We prove that

$$
d(q, \overline{\mathcal{A}})<l_{N}
$$

Set $p_{i}=O^{*}+(0, \ldots, 0, l, 0, \ldots, 0)$ and let $\Pi^{N-1}$ the hyperplane passing through $p_{1}, \ldots, p_{N}$. Since $\overline{\mathcal{A}}$ is convex and $p_{i} \in \overline{\mathcal{A}}$, we obtain that

$$
d(q, \overline{\mathcal{A}})<d\left(q, \Pi^{N-1}\right)<l_{N} .
$$

See Figure 3

Proof of Theorem 3 a) Suppose that $u$ attains its minimum in $\Omega$, and assume $\min _{\Omega} u=0$ and set $\mathcal{C}=\{x \in \Omega: u(x)=0\}$. By contradiction, suppose that the open set $\Omega \backslash \mathcal{C} \neq \emptyset$.

b) Since $\Omega$ is a connected set, there exist $s \in \mathcal{C}$ and $R>0$ such that $B(s, R) \subset \Omega$ and $B(s, R) \cap(\Omega \backslash \mathcal{C}) \neq \emptyset$. Let $p \in B(s, R) \cap(\Omega \backslash \mathcal{C})$. Consider the line $\overline{p s}$. Moving $p$ along this line, we can assume that $B(p, d(p, \mathcal{C})) \subset(\Omega \backslash \mathcal{C})$, and that there exists one point $z \in \mathcal{C}$ such that $d(p, \mathcal{C})=d(p, z)$. 
c) Fix $r$ :

$$
0<r<\frac{d(p, \mathcal{C})}{32(N-1) K^{2}+\frac{7}{8}}
$$

Set

$$
\epsilon(r)=\min \left\{u(z): z \in \overline{B\left(p, d(p, \mathcal{C})-\frac{r}{4}\right)}\right\},
$$

we have that $\epsilon(r)>0$, and we set $\epsilon=\min \{\epsilon(r), r \bar{\xi}\}$.

d) For $r$ and $\epsilon$ as defined in c), consider: $l, l_{N}, \mathcal{A}$ and $v$ as defined in Theorem [4 Without loss of generality, since the set $\mathcal{A}$ is symmetric with respect to both coordinate axis, we can suppose that $\overline{p z}$ belongs to the first quadrant, i.e. that, for every $i=1, \ldots, N, z_{i} \geq p_{i}$, where $z=\left(z_{1}, \ldots, z_{N}\right)$ and $p=\left(p_{1}, \ldots, p_{N}\right)$.

Define the point $q$ on the segment $\overline{p z}$ such that $d(q, p)=d(p, z)-\frac{r}{2}$. Set $q^{*}=q-\left(l, \ldots, l, l_{N}\right), \mathcal{R}\left(q^{*}, l, l_{N}\right)=q^{*}+\mathcal{R}\left(l, l_{N}\right), \mathcal{A}^{*}=q^{*}+\mathcal{A}$ and $v^{*}\left(x+q^{*}\right)=v(x)$. We first claim that

$$
\mathcal{R}\left(q^{*}, l, l_{N}\right) \subset B\left(p, d(p, \mathcal{C})-\frac{r}{4}\right) .
$$

Let $t \in \mathcal{R}\left(q^{*}, l, l_{N}\right)$, then $t$ can be written as $\left(q_{1}-2 \alpha_{1} l, \ldots, q_{N-1}-2 \alpha_{N-1} l, q_{N}-\right.$ $\left.2 \alpha_{N} l_{N}\right)$, with $0 \leq \alpha_{i} \leq 1$, for $i=1, \ldots, N$. Since $r<\frac{d(p, \mathcal{C})}{32(N-1) K^{2}+\frac{7}{8}}$, we have that

$$
\begin{gathered}
d(t, p)^{2}=\sum_{i=1}^{N}\left(q_{i}-2 \alpha_{i} l_{i}-p_{i}\right)^{2}=d(q, p)^{2}+\sum_{i=1}^{N} 4 \alpha_{i}^{2} l_{i}^{2}-\sum_{i=1}^{N} 4 \alpha_{i} l_{i}\left(q_{i}-p_{i}\right) \leq \\
\left(d(p, \mathcal{C})-\frac{r}{2}\right)^{2}+\sum_{i=1}^{N} 4 l_{i}^{2} \leq\left(d(p, \mathcal{C})-\frac{r}{2}\right)^{2}+16(N-1) K^{2} r^{2}+\frac{r^{2}}{4}<\left(d(p, \mathcal{C})-\frac{r}{4}\right)^{2} .
\end{gathered}
$$

See Figure 4

Since $\mathcal{A}^{*} \subset \mathcal{R}\left(q^{*}, l, l_{N}\right)$, we have obtained that

$$
\mathcal{A}^{*} \subset B\left(p, d(p, \mathcal{C})-\frac{r}{4}\right),
$$

so that $u \geq \epsilon$ in $\partial \mathcal{A}^{*}$.

By Lemma 6

we have that

$$
d\left(q, \overline{\mathcal{A}^{*}}\right)<l_{N} \leq \frac{r}{4}
$$

$$
d\left(z, \overline{\mathcal{A}^{*}}\right) \leq d(z, q)+d\left(q, \overline{\mathcal{A}^{*}}\right)<\frac{3}{4} r,
$$

so that

$$
z \in \omega^{*}=B\left(\mathcal{A}^{*}, r\right) \backslash \overline{\mathcal{A}^{*}} .
$$

e) The function $v^{*}$ satisfies

$$
\begin{cases}v^{*} \text { is a weak solution to } F(v) \geq 0 & \text { in } \omega^{*} \\ v^{*}>0 & \text { in } \omega^{*} \\ v^{*}=0 & \text { in } \partial B\left(\mathcal{A}^{*}, r\right) \\ v^{*} \leq \epsilon & \text { in } \partial \mathcal{A}^{*} .\end{cases}
$$

Since $u, v^{*} \in W^{1,2}\left(\omega^{*}\right) \cap C\left(\overline{\omega^{*}}\right), v^{*}$ is a weak subsolution and $u$ is a weak solution to $F(u)=0$, and $v_{\mid \partial \omega^{*}}^{*} \leq u_{\mid \partial \omega^{*}}$, applying Lemma 1 we obtain that $u \geq v^{*}$ in $\omega^{*}$. 


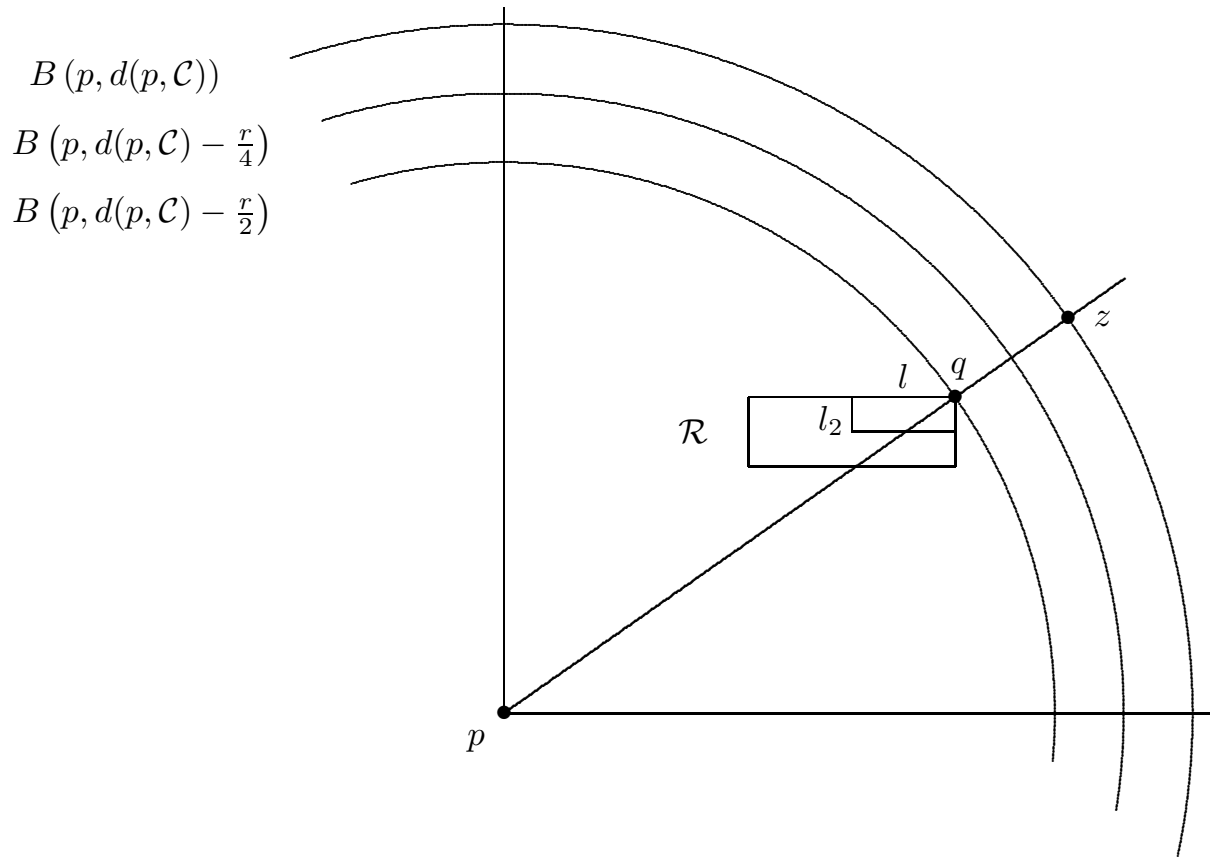

Figure 4 . The set $\mathcal{R}=\mathcal{R}\left(q^{*}, l, l_{2}\right)$ in the case $N=2$.

But $u(z)=0<v^{*}(z)$, a contradiction.

\section{REFERENCES}

[1] H. Berestycki, L. Nirenberg, S.R.S. VAradhan, The principal eigenvalue and maximum principle for second-order elliptic operators in general domains, Communications on Pure and Applied Mathematics 47 (1994), 1, 47-92.

[2] H. Brezis, Analyse fonctionnelle, théorie et applications, Masson, Paris, 1983.

[3] H. Brezis, A. Ponce, Remarks on the strong maximum principle, Differential Integral Equations 16 (2003), 1, 1-12.

[4] A. Cellina On the Strong Maximum Principle, Proc. Amer. Math. Soc. 130 (2001), $2,413-418$.

[5] L. Damascelli, F. Pacella, M. Ramaswamy, A strong maximum principle for a class of non-positone singular elliptic problems, Nonlinear Differential Equations Appl. 10 (2003), 2, 187-196.

[6] J.I. Diaz, Nonlinear partial differential equations and free boundaries, Pitman Research Notes in Mathematics, Vol. 106, 1985.

[7] L. C. Evans, Partial Differential Equations, Graduate Studies in Mathematics, Volume 19, American Mathematical Society, Providence, Rhode Island.

[8] P. Felmer, A. QuAas, On the strong maximum principle for quasilinear elliptic equations and systems, Adv. Differential Equations 7 (2002), 1, 25-46.

[9] D. Gilbarg, N. S. Trudinger, Elliptic Partial Differential Equations of Second Order, Springer-Verlag, Berlin Heidelberg New York Tokyo, 1983.

[10] S. Granlund, Strong maximum principle for a quasi-linear equation with applications, Ann. Acad. Sci. Fenn. Ser. A VI Phys. 21 (1978), 25 pp. 
[11] E. Hopf, Elementare Bemerkungen über die Lsungen partieller Differentialgleichungen zweiter Ordnung vom elliptischen Typus, Sitzungsberichte Preussiche Akademie Wissenschaften, Berlin, 1927, 147-152.

[12] P. Pucci, J. Serrin, The strong maximum principle revisited, J. Differential Equations 196 (2004), 1, 1-66.

[13] P. PuCCI, J. SERRIn, A note on the strong maximum principle for elliptic differential inequalities, J. Math. Pures Appl. (9) 79 (2000), 1, 57-71.

[14] P. PuCCI, J. Serrin, H. Zou, A strong maximum principle and a compact support principle for singular elliptic inequalities, J. Math. Pures Appl. (9) 78 (1999), 8, 769789.

[15] J. SERRIN, On the strong maximum principle for quasilinear second order differential inequalities, J. Functional Anal. 1970, 184-193.

[16] J. Serrin, Commentary on Hopf strong maximum principle, C.S. Morawetz, J.B. Serrin, Y.G. Sinai (Eds.), Selected Works of Eberhard Hopf with commentaries, Amer. Math. Soc., Providence, RI, 2002.

[17] K. TAIRA, A strong maximum principle for degenerate elliptic operators, Comm. Partial Differential Equations 4 (1979), 11, 1201-1212.

[18] J. L. VÁzQUez, A strong maximum principle for some quasilinear elliptic equations, Appl. Math. Optim. 12 (1984), 3, 191-202.

Dipartimento di Matematica e Applicazioni, Universitá degli Studi di Milano-Bicocca, Via R. CozZi 53, 20125 Milano

E-mail address: bertone@matapp.unimib.it

Dipartimento di Matematica e Applicazioni, Universitá degli Studi di Milano-Bicocca, Via R. CozZi 53, 20125 Milano

E-mail address: cellina@matapp.unimib.it

Dipartimento di Matematica e Applicazioni, Universitá degli Studi di Milano-Bicocca, Via R. Cozzi 53, 20125 Milano

E-mail address: elsa@matapp.unimib.it 